\title{
A systematic review on improving implementation of the revitalised integrated disease surveillance and response system in the African region: a health workers' perspective
}

Arthur K. S. Ng'etich ${ }^{1 *}$, Kuku Voyi ${ }^{1}$, Ruth C. Kirinyet ${ }^{2}$, Clifford M. Mutero ${ }^{1,3,4}$

${ }^{1}$ School of Health Systems and Public Health (SHSPH), University of Pretoria, South Africa

${ }^{2}$ School of Public Health, Department of Environmental and Occupational Health, Kenyatta University, Nairobi, Kenya

${ }^{3}$ University of Pretoria Institute for Sustainable Malaria Control (UP ISMC), University of Pretoria, South Africa.

${ }^{4}$ International Centre of Insect Physiology and Ecology, Nairobi, Kenya.

*Corresponding author - Arthur K. S. Ng’etich; arthursaitabau@yahoo.com (AKSN) 


\section{Abstract}

Background: The revised integrated disease surveillance and response (IDSR) guidelines adopted by African member states in 2010 aimed at strengthening surveillance systems critical capacities. Milestones achieved through IDSR strategy implementation prior to adopting the revised guidelines are well documented; however, there is a dearth of knowledge on the progress made post-adoption. This study aimed to review key recommendations resulting from surveillance assessment studies to improve implementation of the revitalised IDSR system in the African region based on health workers' perspectives. The review focused on literature published between 2010 and 2019 post-adopting the revised IDSR guidelines in the African region.

Methods: A systematic literature search in PubMed, Web of Science and Cumulative Index for Nursing and Allied Health Literature was conducted. In addition, manual reference searches and grey literature searches using World Health Organisation Library and Information Networks for Knowledge databases were undertaken. The Preferred Reporting Items for Systematic Reviews and Meta-Analyses statement checklist for systematic reviews was utilised for the review process. Results: Thirty assessment studies met the inclusion criteria. IDSR implementation under the revised guidelines could be improved considerably bearing in mind critical findings and recommendations emanating from the reviewed surveillance assessment studies. Key recommendations alluded to provision of laboratory facilities and improved specimen handling, provision of reporting forms and improved reporting quality, surveillance data accuracy and quality, improved knowledge and surveillance system performance, utilisation of up-to-date information and surveillance system strengthening, provision of resources, enhanced reporting timeliness and completeness, adopting alternative surveillance strategies and conducting further research.

Conclusion: Recommendations on strengthening IDSR implementation in the African region post-adopting the revised guidelines mainly identify surveillance functions focused on reporting, feedback, training, supervision, timeliness and completeness of the surveillance system as aspects requiring policy refinement.

Systematic Review Registration: PROSPERO registration number CRD42019124108. 


\section{Introduction}

Public health surveillance involves continuous collection, analysis and interpretation of health data resulting in timely information dissemination enabling effective public health action [1]. Public health surveillance systems form a critical part of information systems as a key component within the World Health Organization (WHO) health system framework [2]. The International Health Regulations (IHR 2005) within the health system are a legally binding agreement providing a framework to coordinate and manage public health threats [3, 4]. The IHR (2005) necessitated all WHO member states to evaluate ability of their national structures, capacities and resources to achieve effective disease surveillance and response [3]. Prior to IHR (2005), the WHO Regional Office for Africa (WHO-AFRO) and its member states adopted the Integrated Disease Surveillance and Response (IDSR) system [5]. IDSR system framework provided a platform to improve national public health surveillance and response capacities. The IDSR system aims to strengthen the public health system at community, health facility, district, and national levels to ensure timely detection, confirmation and response to public health threats to alleviate illness, disability and mortality [5, 6]. IDSR and IHR frameworks share a common goal of improving timely response to public health events through early detection, notification, verification, response and collaboration activities $[3,6]$. Therefore, member states in WHO African region declared IHR (2005) implementation was to be achieved within the existing IDSR strategy [6]. A review of IDSR guidelines in 2010 was necessary to meet the requirements of disease surveillance and response core capacities strengthening as specified through IHR (2005) implementation by African member states [5-7]. 
The revised IDSR guidelines considered the recommended tools and approaches from IHR (2005) to supplement early warning capabilities in the national surveillance systems and tackle other threats to public health [6]. By 2016, 42 out of 47 countries in the African region had adopted the second edition of the revitalized IDSR technical guidelines [8]. Even though IDSR system adoption by African countries was the most pragmatic approach given resource constraints, there is paucity of knowledge as to the vital recommendations emanating from assessing IDSR system functions [3]. Hence, this review focused on surveillance assessment studies undertaken post-IHR (2005) adoption, which is in line with implementation of the revitalised IDSR system in Africa.

The health workforce across all health system levels are instrumental to achieving effective IDSR system implementation. Hence, giving due consideration to health personnel involvement and their perspectives on full optimisation of surveillance and response systems functionalities is vital to surveillance system improvement. There is insufficient review of literature on evaluation of key policy priorities based on health workers' perspectives ensuing from previous IDSR system assessment studies, which are pertinent to achieving communicable disease control in Africa. Previous systematic reviews have a limited focus on critically assessing fundamental recommendations derived from healthcare workers' perspectives on IDSR system improvement since adopting the revised IDSR guidelines in Africa [9, 10]. Identifying recommendations derived from studies assessing the performance of IDSR system functions is key to focusing decision makers on the critical policy priority areas and guiding implementers towards improving disease surveillance and strengthening the overall health system. However, recommendations to strengthen specific surveillance functions needs to consider the unique nature of the diseases under surveillance. Therefore, the current review aimed to derive key recommendations resulting from 
IDSR system core, support and attribute functions assessment studies to improve implementation of the revitalised IDSR system in the African region based health workers' perspectives.

\section{Research Question}

What lessons can be learned from recommendations derived from previous IDSR system core, support and attribute functions assessment studies to improve implementation of the revitalised IDSR system in the African region based on health workers' perspectives?

The PICO (Population/Interest/Context/Outcome) framework [11, 12] modified to suit qualitative review questions was used to identify keywords in the research question. Therefore, keywords used in the search strategy were derived based on the population comprising of healthcare workers and the phenomenon of interest was the integrated disease surveillance system encompassing core, support and attribute surveillance functions. Furthermore, the review context was Africa and the anticipated outcomes were recommendations to improving surveillance functions based on health workers' perspectives. 


\section{Methods}

A protocol for this systematic review was registered on July 1, 2019 in the International Prospective Register of Systematic Reviews (PROSPERO), registration number CRD42019124108. The review focused on literature published between 2010 and 2019 postrevised IDSR guidelines adoption in Africa. The search included published articles and grey literature for the period between $1^{\text {st }}$ January 2010 to $31^{\text {st }}$ January 2019. Systematic literature searches in PubMed, Web of Science and Cumulative Index for Nursing and Allied Health Literature (CINAHL) using keywords search strategy, in addition to manual reference searches were undertaken. Grey literature searched using World Health Organisation Library and Information Networks for Knowledge (WHOLIS). Each database was searched in consultation with the information specialist of the University of Pretoria Health Faculty Library. Keywords combination using Medical Subject Heading (MeSH) and free text terms relating to the IDSR system were used. The following keywords were used in various combinations ("surveillance", "public health surveillance" [MeSH], "integrated disease surveillance and response", AND “evaluation”, “assessment” AND "health worker", "healthcare personnel” [MeSH], AND “Africa” [MeSH], "Sub Saharan Africa". Individual search terms were combined using the appropriate Boolean operators to generate a list of citations that were saved into Endnote X8 and screened for duplicates.

The review focused on deriving key recommendations based on IDSR system's core and support functions as has been defined by WHO as well as surveillance systems attributes as defined by Centers for Disease Control and Prevention (CDC) $[13,14]$. The inclusion criteria required the literature reviewed be; (1) published full text articles including unpublished studies and grey 
medRxiv preprint doi: https://doi.org/10.1101/2020.09.08.20190553; this version posted September 9, 2020. The copyright holder for this preprint (which was not certified by peer review) is the author/funder, who has granted medRxiv a license to display the preprint in perpetuity.

It is made available under a CC-BY-NC-ND 4.0 International license .

literature for the period between 2010 and 2019; (2) either quantitative or qualitative studies or both assessing implementation of one or more surveillance functions based on health workers' views through interviews and studies involving records reviews or observations; and (3) articles written in English language only. Exclusion criteria considered articles on surveillance assessment studies in countries outside Africa, articles published prior to 2010 before countries adopted the revised IDSR guidelines and articles written in any other language other than English.

\section{Data extraction and synthesis}

All documents and published articles were manually reviewed with duplicates excluded. Preferred Reporting Items for Systematic Reviews and Meta-Analyses (PRISMA) Statement checklist for systematic reviews was utilised in the review process [15]. Data extracted included the country of study, author's name, article publication year, country adoption year of the revised IDSR guidelines, case disease/s of focus, study assessment methodology, surveillance functions assessed, key findings and recommendations. Subsequently, the extracted data was entered into Microsoft Excel prior to analysis. Two reviewers (AKSN, RCK) undertook data extraction and discrepancies between the two resolved by consensus. Data synthesis for quantitative studies was conducted narratively [16]. Analysis of extracted data from qualitative studies was done using thematic synthesis [17]. A matrix of main themes of surveillance functions guided the thematic synthesis with emerging sub-themes. Main themes were based on pre-defined surveillance functions derived from the WHO assessment protocol for national disease surveillance systems and epidemic preparedness, the CDC updated guidelines for evaluating public health surveillance systems and the second edition guidelines for integrated disease surveillance and response in the African region [5, 13, 14]. On the other hand, emerging sub-themes were based on recommendations derived from the reviewed studies. Key recommendations from the reviewed 
studies were identified by first assessing the overall study conclusions then reviewing the study findings that informed the conclusions and recommendations. Reviewers preferred this approach since conclusions emanate from the main study findings, which are linked to critical recommendations that may bear policy implications.

\section{Literature quality appraisal}

Dearholt and Dang's Johns Hopkins Nursing Evidence Appraisal Tool was used for quality appraisal of the reviewed literature. Quality of studies included was based on their strength of evidence (Level I-V) and quality of evidence (Grade A, B \& C) [18]. This was done for each article included in the review by two authors (AKSN, RCK) answering a series of quality appraisal questions independently following which differences were discussed and a consensus reached on quality of literature to be included in the review. The strength of evidence was assigned level I, II, III, IV or V depending on whether the article was based on an experimental study, quasiexperimental study, non-experimental study, nationally recognized experts' opinion based on research evidence or individual expert opinion based on non-research evidence respectively. Furthermore, each included article was assigned grade A, B or C depending on whether the quality of research evidence was of a high, good or low quality respectively [18]. Findings from articles considered to have lower levels of evidence or quality in contrast to findings of higher rated articles were not excluded from this review. However, results from these articles were assessed more critically.

\section{Risk of bias across studies}

Majority of included studies except those supported by document reviews and observations depended on self-reporting by healthcare workers (HCWs). This may have biased their responses towards what they felt was socially desirable at the time of conducting the studies. Secondly, the 
medRxiv preprint doi: https://doi.org/10.1101/2020.09.08.20190553; this version posted September 9, 2020. The copyright holder for this preprint

(which was not certified by peer review) is the author/funder, who has granted medRxiv a license to display the preprint in perpetuity.

It is made available under a CC-BY-NC-ND 4.0 International license.

review focused on assessment studies conducted in the African region, which may have limited the study's perspective from drawing lessons based on IDSR implementation outside Africa. The review only included studies written in English language, which may have introduced some form of selection bias. Lastly, the review was based on extracting relevant studies from four databases; hence limiting the search to what was available in these databases only. 


\section{Results}

\section{Summary of included studies}

The systematic search cumulatively identified 7,491 records from all the databases including a manual reference search. Records retrieved included; 6,244 articles in PubMed, 1,084 articles in Web of Science, 124 articles in CINAHL, 26 grey literature records in WHOLIS and 13 manually searched references as described in the PRISMA flow diagram (Fig 1).

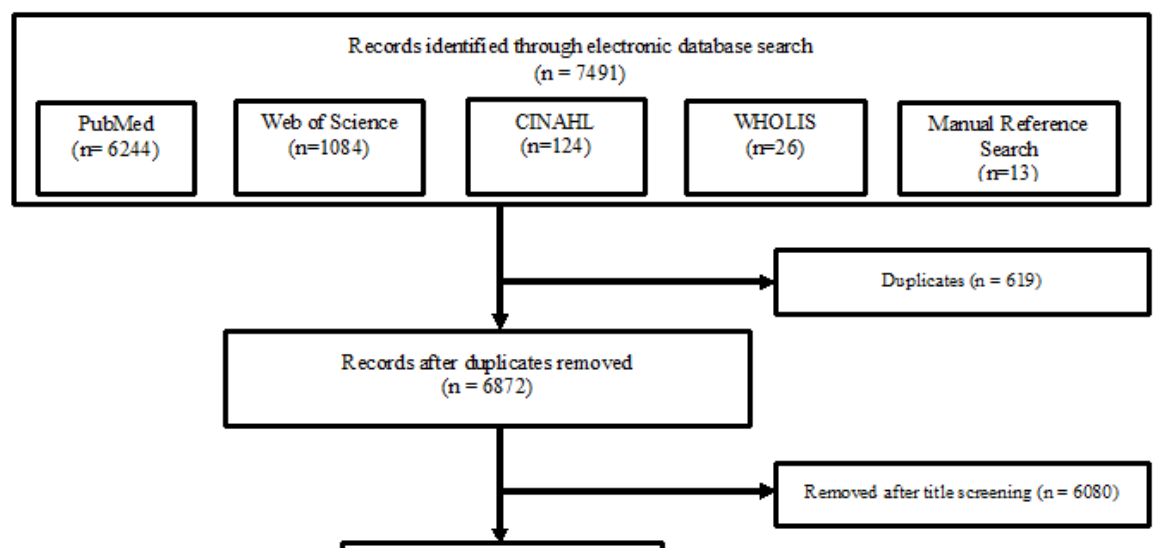

包

Records screened $(\mathrm{n}=792)$
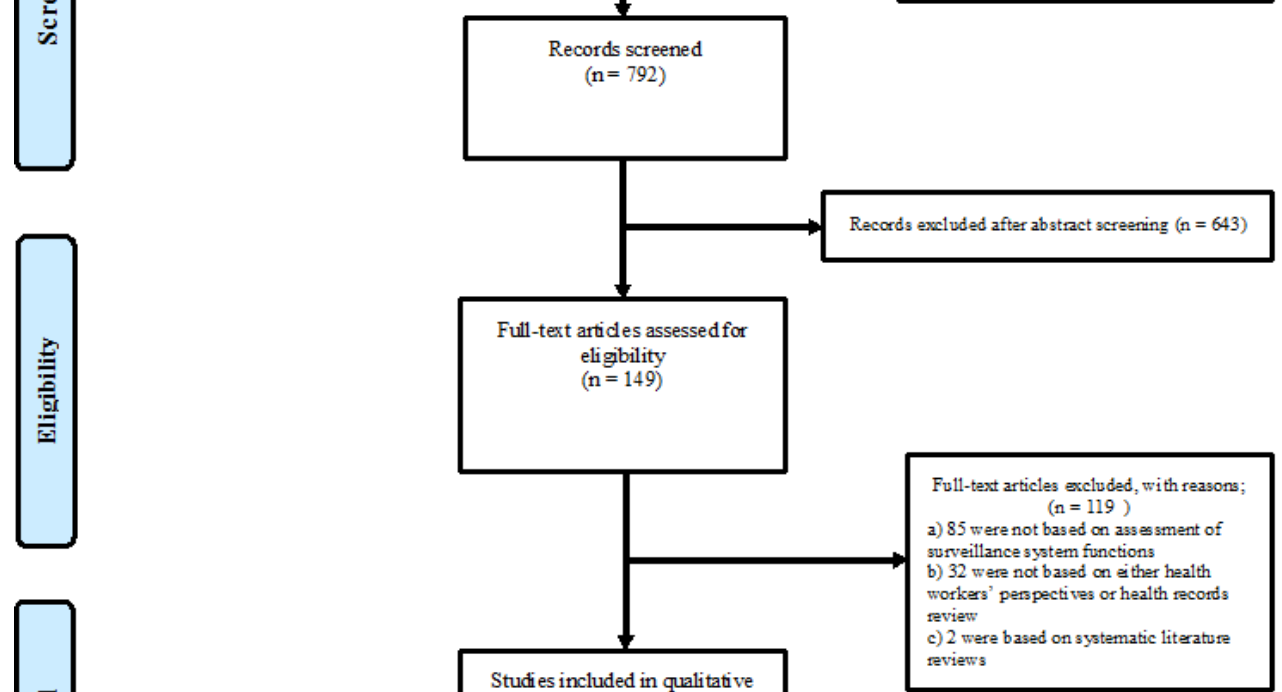

Studi es included in qualitative synthesis

reviews $(\mathrm{n}=30)$

Fig 1. Flow chart summarising the systematic review process 
medRxiv preprint doi: https://doi.org/10.1101/2020.09.08.20190553; this version posted September 9, 2020. The copyright holder for this preprint (which was not certified by peer review) is the author/funder, who has granted medRxiv a license to display the preprint in perpetuity.

It is made available under a CC-BY-NC-ND 4.0 International license .

Abstracts of identified studies were reviewed and the full body text of selected articles read. All identified articles were written in English language. Of the 30 studies meeting the inclusion criteria, 28 (93\%) were assessment studies involving health personnel interviews, 13 (43\%) studies involved a combination of interviews and record reviews while $2(7 \%)$ of the reviewed studies were exclusively based on records review. Surveillance assessment studies were based in 13 countries in the WHO-AFRO region (Ghana, Cameroon, Nigeria, Kenya, Ethiopia, Tanzania, Zimbabwe, Zambia, South Africa, Madagascar, Uganda, Sudan and Malawi). These countries adopted the revitalised IDSR guidelines between 2010 and 2016 [8]. The included assessment studies were based on the revised African IDSR technical guideline disease categories, with twenty studies focused on notifiable diseases requiring immediate reporting while three [19-21] out of the twenty studies mentioned diseases targeted for elimination and eradication including neglected tropical diseases (NTDs) such as guinea worm disease, trachoma and schistosomiasis. However, seven studies did not specify any particular disease in the assessment [22-28]. The reviewed studies covered a combination of surveillance functions with 24 focusing on core functions, 22 on support functions and 18 on surveillance attribute functions. Eighty-seven percent (26/30) of the reviewed studies adopted a cross-sectional study design with the remaining studies adopting either longitudinal [29], retrospective [30, 31] or quasi-experimental [24] study designs. Furthermore, $63 \%$ (29/30) of studies in the review were solely based on quantitative data with two studies exclusively based on qualitative data. However, $30 \%(9 / 30)$ of the reviewed studies involved collection of both types of data. A summary of specific components covered under each of the surveillance function was undertaken, in addition to summarising findings from the reviewed assessment studies (Table 1). Moreover, recommendations to improve IDSR system implementation extracted from the included studies were summarised based on the surveillance 
medRxiv preprint doi: https://doi.org/10.1101/2020.09.08.20190553; this version posted September 9, 2020. The copyright holder for this preprint (which was not certified by peer review) is the author/funder, who has granted medRxiv a license to display the preprint in perpetuity. It is made available under a CC-BY-NC-ND 4.0 International license .

functions (Table 2). Eighteen emerging sub-themes were derived from recommendations specific to four core functions and three support functions (Fig 2). Emerging sub-themes were the identified outcomes of strengthening specific surveillance functions based on the recommendations. Subthemes regarding resources were based on sub-categories of the different resource types. However, no specific sub-themes emerged from the surveillance attributes.

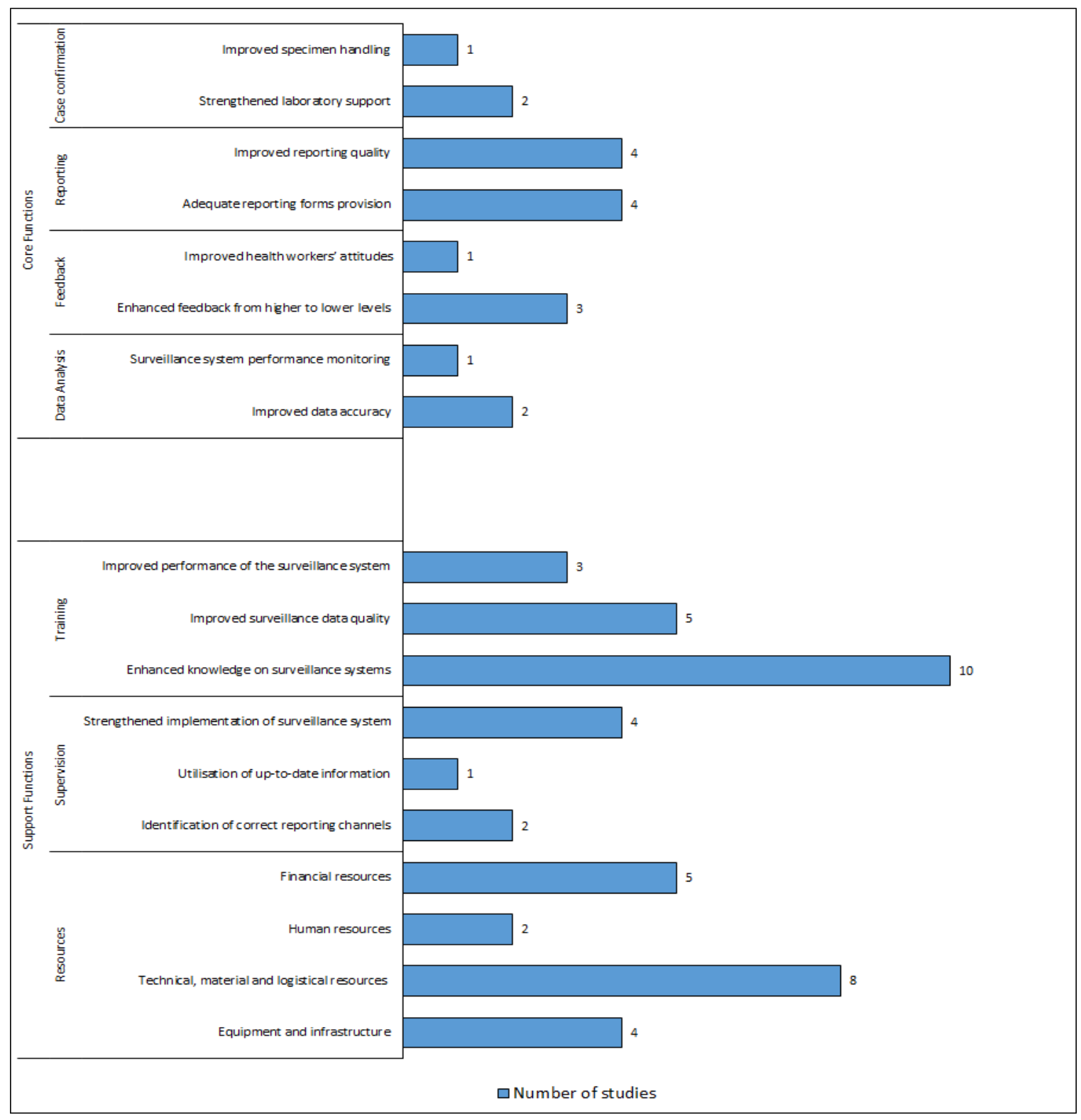

Fig 2. Summary of themes, sub-themes and the number of reviewed studies 
medRxiv preprint doi: https://doi.org/10.1101/2020.09.08.20190553; this version posted September 9, 2020. The copyright holder for this preprint

(which was not certified by peer review) is the author/funder, who has granted medRxiv a license to display the preprint in perpetuity.

It is made available under a CC-BY-NC-ND 4.0 International license.

According to Dearholt and Dang's Johns Hopkins Nursing Evidence Appraisal Tool [18], most studies were assigned level III in terms of evidence strength since 28 out of $30(93 \%)$ of the studies adopted non-experimental study designs (Table 1). In addition, three [28, 32, 33] studies were considered of low quality (Grade C) in terms of evidence quality considering their methodological approach. However, since these studies satisfied the inclusion criteria, they were included in the review and their study findings critically reviewed. 
Table 1. Literature summary and quality appraisal

\begin{tabular}{|c|c|c|c|c|c|c|c|c|c|c|}
\hline No. & Country & $\begin{array}{l}\text { Year } \\
\text { country } \\
\text { adopted } \\
\text { revised } \\
\text { IDSR } \\
\text { guideline }\end{array}$ & $\begin{array}{l}\text { Case } \\
\text { disease/s } \\
\text { mentioned } \\
\text { in the } \\
\text { assessment } \\
\text { study }\end{array}$ & Authors & $\begin{array}{l}\text { Publication } \\
\text { Year }\end{array}$ & $\begin{array}{l}\text { Aim of the } \\
\text { study }\end{array}$ & $\begin{array}{l}\text { Assessment } \\
\text { methodology }\end{array}$ & $\begin{array}{l}\text { Surveillance system } \\
\text { functions assessed }\end{array}$ & Key Findings & $\begin{array}{l}\text { Evidence } \\
\text { Levels(a) } \\
\text { and } \\
\text { Quality } \\
\text { Ratings(b) }^{(b)}\end{array}$ \\
\hline 1. & Nigeria & 2013 & Not specified & $\begin{array}{l}\text { Nnebue et } \\
\text { al.[27] }\end{array}$ & 2013 & $\begin{array}{l}\text { To determine } \\
\text { the } \\
\text { completeness } \\
\text { and timeliness } \\
\text { of data } \\
\text { collection and } \\
\text { disease } \\
\text { notification } \\
\text { To ascertain the } \\
\text { pattern of } \\
\text { transmission of } \\
\text { information } \\
\text { from the health } \\
\text { facility level to } \\
\text { the state level } \\
\\
\text { To come up with } \\
\text { ways in which } \\
\text { the system can } \\
\text { be } \\
\text { improved }\end{array}$ & $\begin{array}{l}\text { Cross-sectional study; } \\
\text { Multistage sampling; } \\
\text { Sample size (270); } \\
\text { Interviews, } \\
\text { observation checklist } \\
\text { and desk review }\end{array}$ & $\begin{array}{l}\text { Core functions: Case } \\
\text { registration, reporting, } \\
\text { feedback } \\
\text { Attributes: Data } \\
\text { accuracy, reporting } \\
\text { completeness and } \\
\text { timeliness }\end{array}$ & $\begin{array}{l}\text {-Health facilities had an } \\
\text { inadequate supply of } \\
\text { IDSR reporting forms } \\
\text {-Surveillance data } \\
\text { reported through the } \\
\text { disease surveillance and } \\
\text { notification system } \\
\text { were not complete and } \\
\text { timely }\end{array}$ & III, B \\
\hline 2. & Nigeria & 2013 & $\begin{array}{l}\text { Diarrhoea, } \\
\text { Measles }\end{array}$ & $\begin{array}{l}\text { Abubakar } \\
\text { et al.[32] }\end{array}$ & 2013 & $\begin{array}{l}\text { To assess the } \\
\text { implementation } \\
\text { of integrated } \\
\text { disease } \\
\text { surveillance and } \\
\text { response in } \\
\text { selected Local } \\
\text { Government } \\
\text { Areas in Kaduna } \\
\text { State. }\end{array}$ & $\begin{array}{l}\text { Cross-sectional } \\
\text { descriptive study; } \\
\text { Multistage sampling; } \\
\text { Interviews, records } \\
\text { and reports review }\end{array}$ & $\begin{array}{l}\text { Core functions: } \\
\text { Reporting, feedback, } \\
\text { data analysis } \\
\text { Support functions: } \\
\text { Standards and } \\
\text { guidelines, resources }\end{array}$ & $\begin{array}{l}\text {-Poor IDSR } \\
\text { implementation } \\
\text {-Insufficient surveillance } \\
\text { resources } \\
\text {-Poor feedback from the } \\
\text { higher to lower levels } \\
\text {-Poor utilisation of } \\
\text { standard case } \\
\text { definitions in health } \\
\text { facilities }\end{array}$ & III, C \\
\hline
\end{tabular}




\begin{tabular}{|c|c|c|c|c|c|c|c|c|c|c|}
\hline 3. & Zimbabwe & 2012 & $\begin{array}{l}\text { Cholera, } \\
\text { Anthrax, } \\
\text { Rabies }\end{array}$ & $\begin{array}{l}\text { Maponga } \\
\text { et al.[34] }\end{array}$ & 2014 & $\begin{array}{l}\text { To evaluate the } \\
\text { notifiable } \\
\text { disease } \\
\text { surveillance } \\
\text { system (NDSS) } \\
\text { in Sanyati } \\
\text { district }\end{array}$ & $\begin{array}{l}\text { Descriptive cross- } \\
\text { sectional study; } \\
\text { Sample size (66); } \\
\text { Interviews }\end{array}$ & $\begin{array}{l}\text { Attributes: } \\
\text { Acceptability, } \\
\text { flexibility, simplicity, } \\
\text { stability, data quality, } \\
\text { timeliness, sensitivity, } \\
\text { representativeness }\end{array}$ & $\begin{array}{l}\text {-Notifiable disease } \\
\text { surveillance system was } \\
\text { useful, acceptable, } \\
\text { simple, sensitive and } \\
\text { timeliness was good } \\
\text {-The system was } \\
\text { threatened by lack of } \\
\text { reporting forms, poor } \\
\text { quality of surveillance } \\
\text { data, low feedback and } \\
\text { lack of knowledge } \\
\text { amongst health workers } \\
\text { on the system } \\
\text {-The time and cost of } \\
\text { transporting reporting } \\
\text { forms to the next level } \\
\text { was unsustainable }\end{array}$ & III, B \\
\hline 4. & Nigeria & 2013 & Not specified & $\begin{array}{l}\text { Nnebue et } \\
\text { al.[26] }\end{array}$ & 2014 & $\begin{array}{l}\text { To determine } \\
\text { the functional } \\
\text { status of the } \\
\text { disease } \\
\text { surveillance and } \\
\text { notification } \\
\text { system and } \\
\text { examine } \\
\text { challenges of } \\
\text { data collection } \\
\text { and disease } \\
\text { notification } \\
\text { across all } \\
\text { surveillance } \\
\text { levels }\end{array}$ & $\begin{array}{l}\text { Descriptive cross- } \\
\text { sectional study; Multi- } \\
\text { sampling technique; } \\
\text { Sample size ( } 270) ; \\
\text { Interviews and } \\
\text { observations }\end{array}$ & $\begin{array}{l}\text { Core functions: } \\
\text { Reporting, feedback } \\
\text { Support functions: } \\
\text { Supervision, training, } \\
\text { resources }\end{array}$ & $\begin{array}{l}\text {-Challenges associated } \\
\text { with disease } \\
\text { surveillance and } \\
\text { notification included; } \\
\text { lack of training, lack of } \\
\text { transportation, poor } \\
\text { motivation, inadequate } \\
\text { supply of forms and } \\
\text { other logistics, poor } \\
\text { funding, ignorance on } \\
\text { the part of the public, } \\
\text { inadequate supervision, } \\
\text { and lack of prompt } \\
\text { feedback }\end{array}$ & III, A \\
\hline 5. & Ghana & 2011 & $\begin{array}{l}\text { Malaria, } \\
\text { HIV/AIDS, } \\
\text { Cholera, } \\
\text { Tuberculosis, } \\
\text { Pneumonia, } \\
\text { Meningitis, } \\
\text { Poliomyelitis } \\
\text { Guinea } \\
\text { Worm } \\
\text { Disease }\end{array}$ & $\begin{array}{l}\text { Adokiya et } \\
\text { al.[21] (a) }\end{array}$ & 2015 & $\begin{array}{l}\text { To evaluate the } \\
\text { IDSR functioning } \\
\text { and its data } \\
\text { quality on the } \\
\text { district health } \\
\text { information } \\
\text { management } \\
\text { system } \\
\text { (DHIMS)in } \\
\text { northern Ghana }\end{array}$ & $\begin{array}{l}\text { Observational study } \\
\text { using mixed methods; } \\
\text { Purposive sampling; } \\
\text { Interviews and reports } \\
\text { review }\end{array}$ & $\begin{array}{l}\text { Core functions: Case } \\
\text { detection, } \\
\text { confirmation, } \\
\text { reporting, data } \\
\text { analysis, epidemic } \\
\text { preparedness and } \\
\text { feedback. }\end{array}$ & $\begin{array}{l}\text {-There are major } \\
\text { challenges to system } \\
\text { functioning including } \\
\text { validity and quality of } \\
\text { data, confirmation } \\
\text { practices of specific } \\
\text { diseases, problems with } \\
\text { supervision and support } \\
\text { at the periphery level, } \\
\text { ill-equipped }\end{array}$ & III, A \\
\hline
\end{tabular}




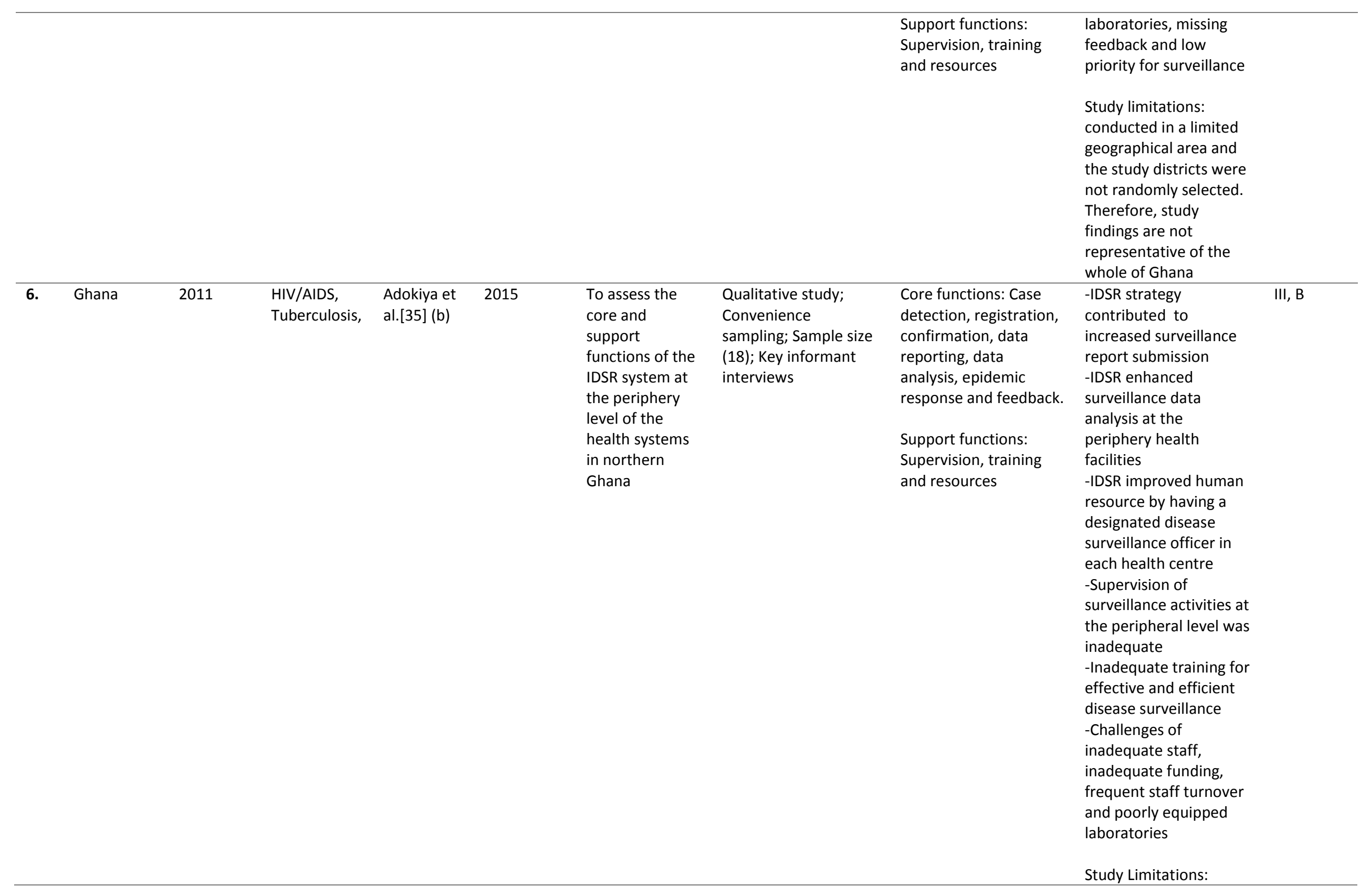




\begin{tabular}{|c|c|c|c|c|c|c|c|c|c|c|}
\hline & & & & & & & & & $\begin{array}{l}\text {-Limited to a small area } \\
\text { in North of Ghana } \\
\text {-Most of the } \\
\text { information was self- } \\
\text { reported and thus } \\
\text { influenced by individual } \\
\text { factors of the few } \\
\text { respondents }\end{array}$ & \\
\hline 7. & Zimbabwe & 2012 & $\begin{array}{l}\text { Malaria, } \\
\text { Rabies, } \\
\text { Polio, } \\
\text { Measles, } \\
\text { Tuberculosis }\end{array}$ & $\begin{array}{l}\text { Tsitsi et } \\
\text { al.[36] }\end{array}$ & 2015 & $\begin{array}{l}\text { To evaluate the } \\
\text { notifiable } \\
\text { disease } \\
\text { surveillance } \\
\text { system (NDSS) } \\
\text { in Beitbridge } \\
\text { district }\end{array}$ & $\begin{array}{l}\text { Descriptive cross- } \\
\text { sectional study; } \\
\text { Purposive sampling; } \\
\text { Sample size (53); } \\
\text { Interviews }\end{array}$ & $\begin{array}{l}\text { Attributes: } \\
\text { Acceptability, } \\
\text { usefulness, flexibility, } \\
\text { simplicity, stability, } \\
\text { sensitivity, data quality, } \\
\text { representativeness and } \\
\text { timeliness }\end{array}$ & $\begin{array}{l}\text {-The surveillance } \\
\text { system was acceptable, } \\
\text { flexible and simple } \\
\text {-The system was } \\
\text { unstable, not sensitive } \\
\text { and not useful } \\
\text {-Reasons for under- } \\
\text { performance of the } \\
\text { surveillance system } \\
\text { were lack of reporting } \\
\text { forms, reporting } \\
\text { guidelines and } \\
\text { knowledge of health } \\
\text { workers on the } \\
\text { surveillance system } \\
\text {-Costs of reporting a } \\
\text { single case of disease } \\
\text { was unreasonably high } \\
\text { using the paper-based } \\
\text { system }\end{array}$ & III, B \\
\hline 8. & Ghana & 2011 & Ebola & $\begin{array}{l}\text { Issah et } \\
\text { al.[29] }\end{array}$ & 2015 & $\begin{array}{l}\text { To assess the } \\
\text { usefulness of } \\
\text { integrated } \\
\text { disease } \\
\text { surveillance and } \\
\text { response on } \\
\text { suspected Ebola } \\
\text { cases }\end{array}$ & $\begin{array}{l}\text { Longitudinal study } \\
\text { design; In-depth } \\
\text { interviews and } \\
\text { documents review }\end{array}$ & $\begin{array}{l}\text { Core functions: Case } \\
\text { detection, case } \\
\text { registration, case } \\
\text { confirmation, } \\
\text { reporting, epidemic } \\
\text { preparedness and } \\
\text { response. } \\
\text { Support functions: } \\
\text { Standards and } \\
\text { guidelines, training, } \\
\text { communication, } \\
\text { coordination, }\end{array}$ & $\begin{array}{l}\text {-Over-dependence by } \\
\text { health staff on } \\
\text { hemorrhage as the only } \\
\text { symptom of EVD and } \\
\text { non-utilisation of case } \\
\text { definitions } \\
\text {-Poor registration of } \\
\text { suspected EVD cases } \\
\text { robbing the system of } \\
\text { vital information for } \\
\text { effective decision- } \\
\text { making }\end{array}$ & II, B \\
\hline
\end{tabular}




\begin{tabular}{|c|c|c|c|c|c|c|c|c|c|c|}
\hline & & & & & & & & $\begin{array}{l}\text { resources, monitoring } \\
\text { and evaluation. } \\
\text { Attributes: } \\
\text { Reporting timeliness }\end{array}$ & $\begin{array}{l}\text {-Good laboratory } \\
\text { support in terms of } \\
\text { timely collection, } \\
\text { transport, testing of } \\
\text { blood samples and } \\
\text { prompt receipt of test } \\
\text { results. } \\
\text {-Inadequate training of } \\
\text { health staff and } \\
\text { community based } \\
\text { surveillance volunteers } \\
\text { on EVD epidemic } \\
\text { preparedness } \\
\text { - Poor practices of blood } \\
\text { sample collection in the } \\
\text { community without } \\
\text { privacy resulting in } \\
\text { stigmatization }\end{array}$ & \\
\hline 9. & Nigeria & 2013 & Not specified & $\begin{array}{l}\text { Lar et } \\
\text { al.[24] }\end{array}$ & 2015 & $\begin{array}{l}\text { Challenges of } \\
\text { IDSR reporting } \\
\text { among } \\
\text { healthcare } \\
\text { personnel in } \\
\text { Mangu, Plateau } \\
\text { State, Nigeria }\end{array}$ & $\begin{array}{l}\text { Quasi-experimental } \\
\text { study; Random } \\
\text { sampling; Sample size } \\
\text { (108); Interviews and } \\
\text { observations }\end{array}$ & $\begin{array}{l}\text { Core functions: } \\
\text { Reporting, feedback } \\
\text { Support functions: } \\
\text { Training }\end{array}$ & $\begin{array}{l}\text {-Mean level knowledge } \\
\text { of the respondents } \\
\text { increased post-training } \\
\text {-There was a statistically } \\
\text { significant association } \\
\text { between training and } \\
\text { reporting form } \\
\text { availability, recognition } \\
\text { of form filling efforts } \\
\text { and feedback }\end{array}$ & II, A \\
\hline 10. & Cameroon & 2011 & Cholera & $\begin{array}{l}\text { Ngwa et } \\
\text { al.[37] }\end{array}$ & 2016 & $\begin{array}{l}\text { To assess the } \\
\text { IDSR strategy } \\
\text { activities in } \\
\text { Cameroon with }\end{array}$ & $\begin{array}{l}\text { Cross-sectional study } \\
\text { design; Sample size } \\
\text { (30), Key informant }\end{array}$ & $\begin{array}{l}\text { Core functions: Case } \\
\text { detection, reporting, } \\
\text { outbreak detection and } \\
\text { feedback }\end{array}$ & $\begin{array}{l}\text {-Lack of mobile phones } \\
\text { in the health facilities } \\
\text { might delay immediate }\end{array}$ & III, B \\
\hline
\end{tabular}




\begin{tabular}{|c|c|c|c|c|c|c|c|c|c|c|}
\hline & & & & & & $\begin{array}{l}\text { a particular } \\
\text { focus on } \\
\text { cholera; } \\
\text { opportunities } \\
\text { and challenges }\end{array}$ & $\begin{array}{l}\text { interviews and } \\
\text { documents review }\end{array}$ & $\begin{array}{l}\text { Support functions: } \\
\text { Standard guidelines, } \\
\text { training, supervision, } \\
\text { resources and } \\
\text { laboratory capacity } \\
\text { Attributes: } \\
\text { Reporting } \\
\text { completeness and } \\
\text { timeliness }\end{array}$ & $\begin{array}{l}\text { reporting of suspected } \\
\text { cases of cholera } \\
\text {-Lack of data analysis } \\
\text { and interpretation at } \\
\text { the health facility and } \\
\text { district levels } \\
\text {-Use of outdated } \\
\text { cholera case definitions } \\
\text {-Health facilities } \\
\text { completely lack } \\
\text { laboratories or have ill- } \\
\text { equipped laboratories } \\
\text { Study limitations: } \\
\text {-Selection bias of focal } \\
\text { persons from only two } \\
\text { regions with frequent } \\
\text { cholera epidemics out } \\
\text { of the ten regions }\end{array}$ & \\
\hline 11. & South Africa & 2013 & $\begin{array}{l}33 \text { notifiable } \\
\text { conditions in } \\
\text { South Africa } \\
\text { (among } \\
\text { them are } \\
\text { diseases } \\
\text { targeted for } \\
\text { elimination } \\
\text { such as } \\
\text { Trachoma) }\end{array}$ & $\begin{array}{l}\text { Benson et } \\
\text { al.[20] }\end{array}$ & 2016 & $\begin{array}{l}\text { To determine } \\
\text { the perceptions } \\
\text { of key } \\
\text { stakeholders on } \\
\text { the national } \\
\text { notifiable } \\
\text { disease } \\
\text { surveillance } \\
\text { system } \\
\text { attributes }\end{array}$ & $\begin{array}{l}\text { Cross-sectional survey; } \\
\text { Interviews }\end{array}$ & $\begin{array}{l}\text { Attributes: } \\
\text { Acceptability, } \\
\text { flexibility, simplicity, } \\
\text { timeliness and } \\
\text { usefulness }\end{array}$ & $\begin{array}{l}\text {-The majority of key } \\
\text { stakeholders scored the } \\
\text { NDSS as low on the } \\
\text { attributes of } \\
\text { acceptability, flexibility } \\
\text { and usefulness. } \\
\text {-Factors associated with } \\
\text { key stakeholders } \\
\text { perceptions were years } \\
\text { of experience, NDSS } \\
\text { training, age below } 35 \\
\text { years, participation in } \\
\text { disease detection and } \\
\text { national outbreak } \\
\text { response team }\end{array}$ & III, B \\
\hline & Kenya & 2012 & $\begin{array}{l}35 \text { priority } \\
\text { diseases as } \\
\text { provided in } \\
\text { the IDSR } \\
\text { technical } \\
\text { guideline }\end{array}$ & $\begin{array}{l}\text { Mwatondo } \\
\text { et al.[38] }\end{array}$ & 2016 & $\begin{array}{l}\text { To determine } \\
\text { the prevalence } \\
\text { of adequate } \\
\text { reporting and } \\
\text { factors } \\
\text { associated with } \\
\text { IDSR reporting }\end{array}$ & $\begin{array}{l}\text { Cross-sectional survey; } \\
\text { Stratified random } \\
\text { sampling; Sample size } \\
(183) ; \text { Interviews and } \\
\text { reports review }\end{array}$ & $\begin{array}{l}\text { Core functions: } \\
\text { Reporting } \\
\text { Support functions: } \\
\text { Standards and } \\
\text { guidelines (i.e. case } \\
\text { definitions), resources }\end{array}$ & $\begin{array}{l}\text {-Sub-optimal reporting } \\
\text { of IDSR priority diseases } \\
\text { in a majority of health } \\
\text { facilities } \\
\text {-Health facilities } \\
\text { submitted complete } \\
\text { and timely reports }\end{array}$ & III, A \\
\hline
\end{tabular}




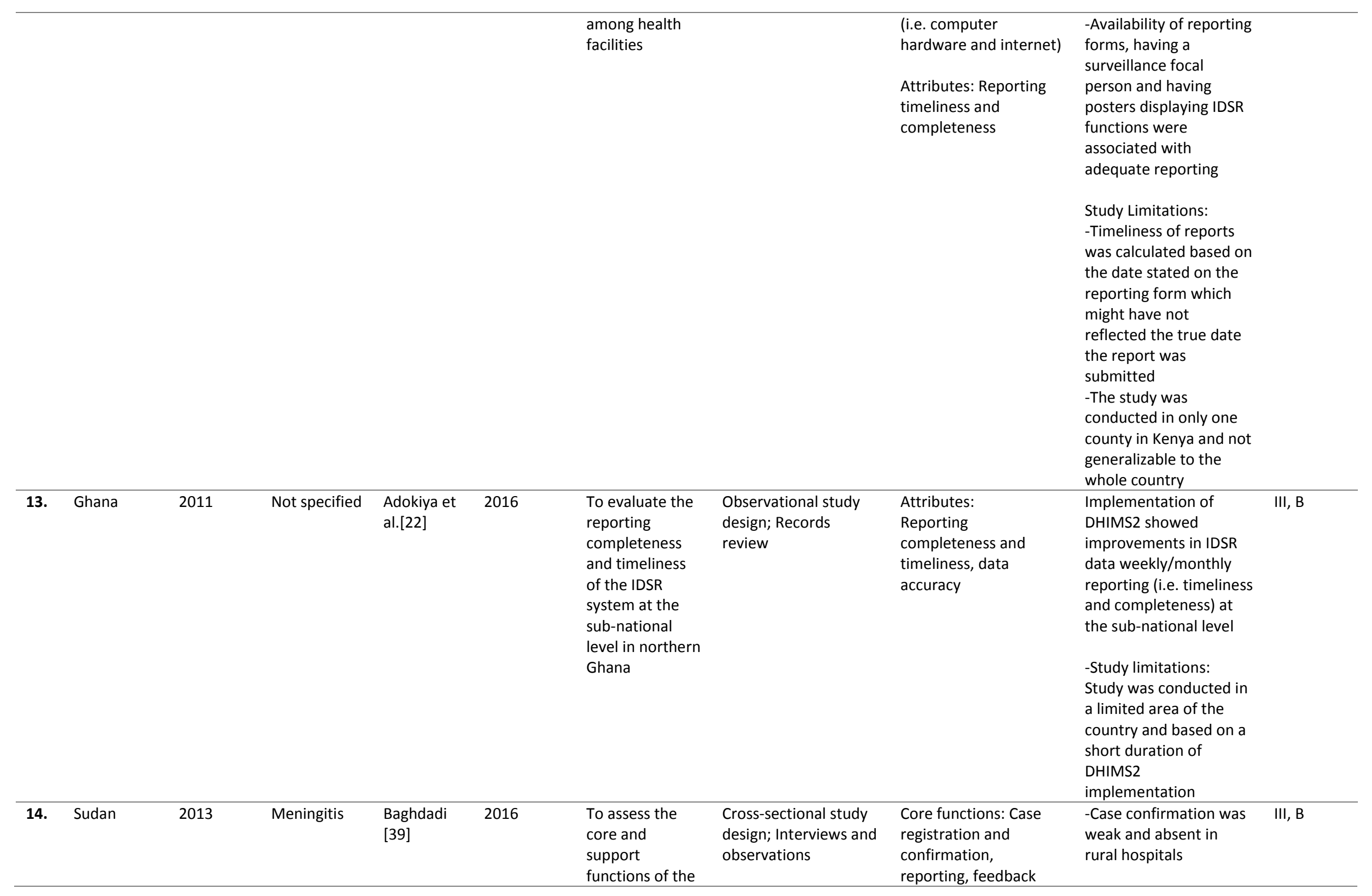




\begin{tabular}{|c|c|c|c|c|c|c|c|c|c|c|}
\hline & & & & & & $\begin{array}{l}\text { surveillance } \\
\text { system with } \\
\text { regards to } \\
\text { meningitis }\end{array}$ & & $\begin{array}{l}\text { Support functions: } \\
\text { Standards and } \\
\text { guidelines (case } \\
\text { definitions), training, } \\
\text { laboratory capacity, } \\
\text { communication } \\
\text { facilities }\end{array}$ & $\begin{array}{l}\text {-52\% of the hospitals } \\
\text { had health personnel } \\
\text { trained on surveillance } \\
-42 \% \text { of the hospitals } \\
\text { received feedback } \\
\text { reports from their } \\
\text { localities } \\
\text { Study limitations } \\
\text {-Limited to hospitals } \\
\text { only } \\
\text {-Not all hospitals were } \\
\text { visited physically } \\
\text {-Dependency on } \\
\text { telephone interviews } \\
\text {-Not all core and } \\
\text { support functions were } \\
\text { assessed }\end{array}$ & \\
\hline & Ghana & 2011 & Ebola & $\begin{array}{l}\text { Adokiya } \\
\text { and } \\
\text { Awoonor- } \\
\text { Williams } \\
{[40]}\end{array}$ & 2016 & $\begin{array}{l}\text { To assess the } \\
\text { Ebola Virus } \\
\text { Disease } \\
\text { surveillance and } \\
\text { response system } \\
\text { in northern } \\
\text { Ghana }\end{array}$ & $\begin{array}{l}\text { Observational cross- } \\
\text { sectional study design; } \\
\text { Sample size (47); } \\
\text { Interviews }\end{array}$ & $\begin{array}{l}\text { Core functions: Case } \\
\text { detection and } \\
\text { confirmation, } \\
\text { reporting, feedback, } \\
\text { outbreak preparedness } \\
\text { Support functions: } \\
\text { supervision, training, } \\
\text { resources }\end{array}$ & $\begin{array}{l}\text {-Lack of registers for } \\
\text { Ebola data } \\
\text {-Inadequate Personal } \\
\text { Protective Equipment } \\
\text { (PPEs) } \\
\text {-Inadequate laboratory } \\
\text { capacity } \\
\text {-Delays in specimen } \\
\text { taking }\end{array}$ & III, B \\
\hline & Zimbabwe & 2012 & Typhoid & $\begin{array}{l}\text { Mairosi et } \\
\text { al.[41] }\end{array}$ & 2016 & $\begin{array}{l}\text { To evaluate the } \\
\text { notifiable } \\
\text { disease } \\
\text { surveillance } \\
\text { system (NDSS) } \\
\text { in Centenary, } \\
\text { Zimbabwe by } \\
\text { assessing the } \\
\text { usefulness of } \\
\text { the NDSS, the } \\
\text { systems } \\
\text { attributes, } \\
\text { health care } \\
\text { worker }\end{array}$ & $\begin{array}{l}\text { Descriptive cross } \\
\text { sectional study design; } \\
\text { Purposive sampling; } \\
\text { Sample size (59); } \\
\text { Interviews and records } \\
\text { review }\end{array}$ & $\begin{array}{l}\text { Core functions: } \\
\text { Reporting } \\
\text { Attributes: } \\
\text { Usefulness, simplicity, } \\
\text { acceptability, stability, } \\
\text { flexibility sensitivity, } \\
\text { data quality and } \\
\text { timeliness }\end{array}$ & $\begin{array}{l}\text {-Low levels of } \\
\text { knowledge on notifiable } \\
\text { disease surveillance } \\
\text { system resulting to } \\
\text { missing notifiable } \\
\text { diseases, } \\
\text { underreporting and } \\
\text { poor case management } \\
\text {-The notifiable disease } \\
\text { surveillance system was } \\
\text { unstable and lacked } \\
\text { sensitivity }\end{array}$ & III, B \\
\hline
\end{tabular}




\begin{tabular}{|c|c|c|c|c|c|c|c|c|c|c|}
\hline & & & & & & $\begin{array}{l}\text { knowledge } \\
\text { levels and cost } \\
\text { of running the } \\
\text { system }\end{array}$ & & & & \\
\hline 17. & Nigeria & 2013 & Not specified & $\begin{array}{l}\text { Iwu et } \\
\text { al.[25] }\end{array}$ & 2016 & $\begin{array}{l}\text { To identify gaps } \\
\text { in disease } \\
\text { reporting } \\
\text { among health } \\
\text { care workers } \\
\text { within a } \\
\text { resource limited } \\
\text { setting in order } \\
\text { to develop } \\
\text { programs that } \\
\text { improve their } \\
\text { knowledge, } \\
\text { attitude and } \\
\text { practices }\end{array}$ & $\begin{array}{l}\text { Descriptive cross- } \\
\text { sectional design; } \\
\text { Stratified simple } \\
\text { random sampling; } \\
\text { Sample size (449); } \\
\text { Interviews and } \\
\text { observations }\end{array}$ & $\begin{array}{l}\text { Core functions: } \\
\text { Reporting } \\
\text { Support functions: } \\
\text { Training, resources }\end{array}$ & $\begin{array}{l}\text {-Most respondents } \\
\text { perceived inadequate } \\
\text { training, lack of } \\
\text { equipment and } \\
\text { inadequate supply of } \\
\text { reporting forms as } \\
\text { limiting factors to } \\
\text { disease reporting }\end{array}$ & III, A \\
\hline 18. & Ethiopia & 2010 & Not specified & $\begin{array}{l}\text { Begashaw } \\
\text { and } \\
\text { Tesfaye } \\
\text { [28] }\end{array}$ & 2016 & $\begin{array}{l}\text { To assess the } \\
\text { implementation } \\
\text { of integrated } \\
\text { disease } \\
\text { surveillance and } \\
\text { response in } \\
\text { selected health } \\
\text { facilities in } \\
\text { Dawuro Zone }\end{array}$ & $\begin{array}{l}\text { Descriptive cross } \\
\text { sectional facility-based } \\
\text { study; Multi stage } \\
\text { sampling; Interviews }\end{array}$ & $\begin{array}{l}\text { Core functions: } \\
\text { Reporting, feedback, } \\
\text { data analysis, } \\
\text { Support functions: } \\
\text { Resources }\end{array}$ & $\begin{array}{l}\text {-Majority of health } \\
\text { facilities had no form of } \\
\text { data analysis available } \\
\text {-The available } \\
\text { surveillance resources } \\
\text { such as bicycles, } \\
\text { motorcycles, computers } \\
\text { and printers were non- } \\
\text { functional in most } \\
\text { health facilities } \\
\text {-Feedback reports from } \\
\text { the regional level to the } \\
\text { district level and from } \\
\text { the zone to the health } \\
\text { centers were } \\
\text { unavailable }\end{array}$ & III, C \\
\hline 19. & South Africa & 2013 & $\begin{array}{l}\text { Measles, } \\
\text { Meningoccal } \\
\text { Meningitis, } \\
\text { Typhoid }\end{array}$ & $\begin{array}{l}\text { Benson et } \\
\text { al.[30] }\end{array}$ & 2017 & $\begin{array}{l}\text { To compare } \\
\text { laboratory } \\
\text { surveillance } \\
\text { with the } \\
\text { notifiable } \\
\text { diseases } \\
\text { surveillance } \\
\text { system (NDSS) }\end{array}$ & $\begin{array}{l}\text { Retrospective study } \\
\text { design; Records } \\
\text { review }\end{array}$ & $\begin{array}{l}\text { Attributes: Data } \\
\text { quality, stability, } \\
\text { representativeness, } \\
\text { sensitivity and positive } \\
\text { predictive value }\end{array}$ & $\begin{array}{l}\text {-Laboratory data } \\
\text { completeness was } \\
\text { higher for measles and } \\
\text { meningitis but not for } \\
\text { typhoid } \\
\text {-Both stability and } \\
\text { representativeness was } \\
\text { higher for laboratory }\end{array}$ & III, A \\
\hline
\end{tabular}




\begin{tabular}{|c|c|c|c|c|c|c|c|c|c|c|}
\hline & & & & & & & & & $\begin{array}{l}\text { compared to notified } \\
\text { measles, meningitis and } \\
\text { typhoid }\end{array}$ & \\
\hline 20. & Zambia & 2012 & $\begin{array}{l}\text { Dysentery, } \\
\text { Malaria, HIV, } \\
\text { Tuberculosis, } \\
\text { Typhoid, } \\
\text { Measles }\end{array}$ & $\begin{array}{l}\text { Mandyata } \\
\text { et al.[42] }\end{array}$ & 2017 & $\begin{array}{l}\text { To investigate } \\
\text { and report on } \\
\text { the existing } \\
\text { challenges in } \\
\text { the } \\
\text { implementation } \\
\text { of the IDSR } \\
\text { strategy in a } \\
\text { resource limited } \\
\text { country from a } \\
\text { health worker } \\
\text { perspective }\end{array}$ & $\begin{array}{l}\text { Qualitative study } \\
\text { design; Purposive } \\
\text { sampling; Key } \\
\text { informant interviews }\end{array}$ & $\begin{array}{l}\text { Core functions: Case } \\
\text { detection, } \\
\text { confirmation, } \\
\text { registration, reporting, } \\
\text { data analysis, response } \\
\text { and control, feedback. } \\
\text { Support functions: } \\
\text { Training, logistical } \\
\text { support, supervision. } \\
\text { Attributes: } \\
\text { Representativeness, } \\
\text { stability }\end{array}$ & $\begin{array}{l}\text {-Major successes } \\
\text { included } \\
\text { operationalised } \\
\text { epidemic preparedness } \\
\text { and response plans, full } \\
\text {-time staff, dedicated } \\
\text { disease surveillance } \\
\text { budgets and adoption } \\
\text { of IDSR technical } \\
\text { guidelines across all } \\
\text { levels } \\
\text {-Major challenges } \\
\text { included inadequate } \\
\text { trained human } \\
\text { resource, poor } \\
\text { infrastructure and } \\
\text { coordination challenges. } \\
\text { Study limitation: } \\
\text {-The study findings from } \\
\text { two conveniently } \\
\text { sampled districts were } \\
\text { perceived to be } \\
\text { transferable to other } \\
\text { districts throughout the } \\
\text { country. } \\
\text {-Only certain key areas } \\
\text { from each of the four } \\
\text { components of the IDSR } \\
\text { strategy were selected } \\
\text { to highlight some of the } \\
\text { main challenges }\end{array}$ & III, B \\
\hline 21. & Tanzania & 2011 & Malaria & $\begin{array}{l}\text { Mboera et } \\
\text { al.[33] }\end{array}$ & 2017 & $\begin{array}{l}\text { To assess } \\
\text { malaria } \\
\text { surveillance } \\
\text { system at the } \\
\text { facility and } \\
\text { district levels in } \\
\text { order to identify }\end{array}$ & $\begin{array}{l}\text { Cross-sectional study } \\
\text { design; Purposive } \\
\text { sampling; Sample size } \\
\text { (20); In-depth } \\
\text { interviews, } \\
\text { observations and } \\
\text { documents review }\end{array}$ & $\begin{array}{l}\text { Core functions: Case } \\
\text { registration, reporting, } \\
\text { data analysis, } \\
\text { response, feedback, } \\
\text { Support functions: } \\
\text { Standards and }\end{array}$ & $\begin{array}{l}\text {-Poor data } \\
\text { management, } \\
\text { inefficient reporting, } \\
\text { shortage of data } \\
\text { collection and } \\
\text { processing tools, limited } \\
\text { data analysis capacity, }\end{array}$ & III, C \\
\hline
\end{tabular}




\begin{tabular}{|c|c|c|c|c|c|c|c|c|c|c|}
\hline & & & & & & $\begin{array}{l}\text { key barriers, } \\
\text { constraints and } \\
\text { priority actions } \\
\text { for malaria } \\
\text { surveillance } \\
\text { strengthening } \\
\text { To explore the } \\
\text { use of evidence } \\
\text { in health } \\
\text { planning and } \\
\text { decision making } \\
\text { at the facility } \\
\text { and district } \\
\text { levels }\end{array}$ & & $\begin{array}{l}\text { guidelines, training, } \\
\text { resources, } \\
\text { communication, } \\
\text { Attributes: Reporting } \\
\text { timeliness and } \\
\text { completeness, } \\
\text { usefulness, }\end{array}$ & $\begin{array}{l}\text { over-burdened health } \\
\text { staff, weak } \\
\text { communication } \\
\text { systems, weak capacity } \\
\text { for facility level decision } \\
\text { making, multiple } \\
\text { surveillance systems } \\
\text { demanded by different } \\
\text { vertical programmes }\end{array}$ & \\
\hline 22. & Ethiopia & 2010 & $\begin{array}{l}\text { Vaccine } \\
\text { Preventable } \\
\text { Diseases i.e. } \\
\text { Acute Flaccid } \\
\text { Paralysis, } \\
\text { Measles and } \\
\text { Neonatal } \\
\text { Tetanus }\end{array}$ & $\begin{array}{l}\text { Lakew et } \\
\text { al.[43] }\end{array}$ & 2017 & $\begin{array}{l}\text { To assess the } \\
\text { performances of } \\
\text { disease } \\
\text { surveillance and } \\
\text { routine } \\
\text { immunization } \\
\text { with particular } \\
\text { focus on vaccine } \\
\text { preventable } \\
\text { diseases (VPD) } \\
\text { in Amhara } \\
\text { Region, Ethiopia }\end{array}$ & $\begin{array}{l}\text { Cross-sectional study } \\
\text { design; Purposive } \\
\text { sampling; Interviews, } \\
\text { observations and } \\
\text { documents review }\end{array}$ & $\begin{array}{l}\text { Core functions: Case } \\
\text { confirmation, } \\
\text { reporting, evaluation } \\
\text { Support functions: } \\
\text { Supervision, training, } \\
\text { surveillance guidelines } \\
\text { and case definitions, } \\
\text { coordination }\end{array}$ & $\begin{array}{l}\text {-Less than half of the } \\
\text { health offices and } \\
\text { health facilities had } \\
\text { clearly written and } \\
\text { communicated terms of } \\
\text { reference and standard } \\
\text { operating procedures } \\
\text { for their surveillance } \\
\text { focal persons. } \\
\text {-Active case searches } \\
\text { were hardly being } \\
\text { conducted by } \\
\text { surveillance focal } \\
\text { persons. } \\
\text {-Low documentation } \\
\text { and incomplete case } \\
\text { files of reported } \\
\text { diseases } \\
\text {-Involvement of } \\
\text { laboratories in } \\
\text { surveillance activities at } \\
\text { all levels was limited }\end{array}$ & III, A \\
\hline 23. & Zambia & 2012 & Not specified & $\begin{array}{l}\text { Haakonde } \\
\text { et al.[23] }\end{array}$ & 2018 & $\begin{array}{l}\text { To assess the } \\
\text { factors affecting } \\
\text { the } \\
\text { implementation } \\
\text { of the IDSR in }\end{array}$ & $\begin{array}{l}\text { Descriptive cross- } \\
\text { sectional facility-based } \\
\text { study design; } \\
\text { Convenient sampling; }\end{array}$ & $\begin{array}{l}\text { Core functions: } \\
\text { Reporting, feedback } \\
\text { Support functions: } \\
\text { Training, supervision, }\end{array}$ & $\begin{array}{l}\text {-IDSR lacked local } \\
\text { support in terms of } \\
\text { periodical training and } \\
\text { mentorship, regular and } \\
\text { scheduled supervisory }\end{array}$ & III, B \\
\hline
\end{tabular}




\begin{tabular}{|c|c|c|c|c|c|c|c|c|c|c|}
\hline & & & & & & $\begin{array}{l}\text { public health } \\
\text { care facilities in } \\
\text { Rufunsa District, } \\
\text { Zambia. }\end{array}$ & $\begin{array}{l}\text { Sample size (34); } \\
\text { Interviews }\end{array}$ & $\begin{array}{l}\text { resources (logistical, } \\
\text { financial, equipment) }\end{array}$ & $\begin{array}{l}\text { assistance and financial } \\
\text { aid across surveillance } \\
\text { levels. } \\
\text {-Lack of prompt } \\
\text { feedback from higher } \\
\text { levels. } \\
\text {-Lack of adequate } \\
\text { coordination and } \\
\text { communication across } \\
\text { surveillance levels. }\end{array}$ & \\
\hline 24. & Malawi & 2014 & $\begin{array}{l}\text { Ebola, } \\
\text { Tuberculosis, } \\
\text { Malaria }\end{array}$ & $\begin{array}{l}\text { Wu et } \\
\text { al.[44] }\end{array}$ & 2018 & $\begin{array}{l}\text { To describe the } \\
\text { process of case } \\
\text { identification } \\
\text { and reporting in } \\
\text { practice, } \\
\text { and explore the } \\
\text { differences } \\
\text { between the } \\
\text { IDSR guideline } \\
\text { and actual } \\
\text { implementation } \\
\text { using } \\
\text { timeliness and } \\
\text { completeness as } \\
\text { key indicators to } \\
\text { evaluate IDSR } \\
\text { performance in } \\
\text { Malawi }\end{array}$ & $\begin{array}{l}\text { Mixed methods study } \\
\text { design; Key informant } \\
\text { interviews, focus } \\
\text { groups and reports } \\
\text { review }\end{array}$ & $\begin{array}{l}\text { Core functions: Case } \\
\text { detection, Reporting } \\
\text { Support functions: } \\
\text { Standard case } \\
\text { definitions, Laboratory } \\
\text { capacity, Training } \\
\text { Supervision, Resources } \\
\text { Attributes: Reporting } \\
\text { completeness and } \\
\text { timeliness }\end{array}$ & $\begin{array}{l}\text {-Differences between } \\
\text { IDSR technical guideline } \\
\text { and actual practice } \\
\text { existed } \\
\text {-System shortcomings } \\
\text { resulted from financial } \\
\text { constraints and poor } \\
\text { infrastructure }\end{array}$ & III, A \\
\hline 25. & Nigeria & 2013 & $\begin{array}{l}\text { Cholera, } \\
\text { Gastroenteri } \\
\text { tis, Measles, } \\
\text { Typhoid } \\
\text { fever, } \\
\text { Schistosomia } \\
\text { sis }\end{array}$ & $\begin{array}{l}\text { Dairo et } \\
\text { al.[19] }\end{array}$ & 2018 & $\begin{array}{l}\text { To assess } \\
\text { compliance with } \\
\text { the surveillance } \\
\text { and response } \\
\text { guidelines for } \\
\text { epidemic-prone } \\
\text { diseases }\end{array}$ & $\begin{array}{l}\text { Descriptive cross- } \\
\text { sectional study design; } \\
\text { Multi stage sampling; } \\
\text { Sample size (198); } \\
\text { Interviews, } \\
\text { observations and } \\
\text { records review }\end{array}$ & $\begin{array}{l}\text { Core functions: Case } \\
\text { detection, case } \\
\text { confirmation, case } \\
\text { registration, reporting, } \\
\text { feedback, data } \\
\text { analysis, epidemic } \\
\text { preparedness } \\
\text { Support functions: } \\
\text { Standard case } \\
\text { definitions, } \\
\text { supervision, resources }\end{array}$ & $\begin{array}{l}\text {-Inadequacy of } \\
\text { laboratory support for } \\
\text { surveillance activities at } \\
\text { the local levels }\end{array}$ & III, B \\
\hline
\end{tabular}




\begin{tabular}{|c|c|c|c|c|c|c|c|c|c|c|}
\hline 26. & Madagascar & 2013 & $\begin{array}{l}\text { Malaria, } \\
\text { Diarrhoea, } \\
\text { Acute } \\
\text { Respiratory } \\
\text { Infections, } \\
\text { Measles, } \\
\text { Acute Flaccid } \\
\text { Paralysis, } \\
\text { Chikungunya }\end{array}$ & $\begin{array}{l}\text { Randriami- } \\
\text { arana et } \\
\text { al.[45] }\end{array}$ & 2018 & $\begin{array}{l}\text { To evaluate } \\
\text { performance of } \\
\text { the reinforced } \\
\text { integrated } \\
\text { disease } \\
\text { surveillance and } \\
\text { response } \\
\text { strategy using } \\
\text { attributes and } \\
\text { technological } \\
\text { assessment }\end{array}$ & $\begin{array}{l}\text { Evaluation study } \\
\text { design; Random } \\
\text { sampling; Interviews }\end{array}$ & $\begin{array}{l}\text { Support functions: } \\
\text { Standard and } \\
\text { guidelines, resources } \\
\text { (infrastructure) } \\
\text { Attributes: Simplicity, } \\
\text { data quality, } \\
\text { completeness and } \\
\text { timeliness }\end{array}$ & $\begin{array}{l}\text {-Short Message Service } \\
\text { (SMS) improved IDSR } \\
\text { data completeness but } \\
\text { there is still a challenge } \\
\text { with timeliness and } \\
\text { data quality }\end{array}$ & III, A \\
\hline 27. & Uganda & 2012 & $\begin{array}{l}\text { Cholera, } \\
\text { Polio }\end{array}$ & $\begin{array}{l}\text { Masiira et } \\
\text { al.[46] }\end{array}$ & 2019 & $\begin{array}{l}\text { To present } \\
\text { findings from an } \\
\text { assessment of } \\
\text { IDSR core } \\
\text { activities and } \\
\text { support } \\
\text { functions five } \\
\text { years following } \\
\text { implementation } \\
\text { of the } \\
\text { revitalized IDSR } \\
\text { programme }\end{array}$ & $\begin{array}{l}\text { Cross sectional survey; } \\
\text { Purposive sampling; } \\
\text { Sample size (202); } \\
\text { Interviews, focus } \\
\text { groups and } \\
\text { observations }\end{array}$ & $\begin{array}{l}\text { Core functions: Case } \\
\text { detection, case } \\
\text { registration, case } \\
\text { confirmation, } \\
\text { reporting, feedback, } \\
\text { data analysis, epidemic } \\
\text { preparedness and } \\
\text { response } \\
\text { Support functions: } \\
\text { Standard case } \\
\text { definitions, training, } \\
\text { resources, } \\
\text { Attributes: Reporting } \\
\text { completeness and } \\
\text { timeliness }\end{array}$ & $\begin{array}{l}\text {-Inadequate number of } \\
\text { trained frontline health } \\
\text { workers } \\
\text {-Inadequate funding to } \\
\text { support IDSR activities } \\
\text { at district and health } \\
\text { facility levels } \\
\text {-Poor perception of } \\
\text { IDSR as a vertical } \\
\text { programme by some } \\
\text { health workers } \\
\text {-Irregular supervision } \\
\text {-High turnover of } \\
\text { trained health } \\
\text { workers }\end{array}$ & III, A \\
\hline 28. & Nigeria & 2013 & Measles & $\begin{array}{l}\text { Ameh et al. } \\
\text { [31] }\end{array}$ & 2016 & $\begin{array}{l}\text { To evaluate the } \\
\text { case-based } \\
\text { measles } \\
\text { surveillance } \\
\text { system }\end{array}$ & $\begin{array}{l}\text { Evaluation study; } \\
\text { Retrospective records } \\
\text { review; Interviews }\end{array}$ & $\begin{array}{l}\text { Core functions: Case } \\
\text { detection, case } \\
\text { confirmation } \\
\text { Attributes: Positive } \\
\text { predictive value, data } \\
\text { quality, acceptability, } \\
\text { stability, } \\
\text { representativeness, } \\
\text { usefulness, timeliness, }\end{array}$ & $\begin{array}{l}\text {-Proportion of measles } \\
\text { laboratory results } \\
\text { availed exceeded WHO } \\
\text { recommended target } \\
\text {-Reporting timelines } \\
\text { and positive predictive } \\
\text { value for cased based } \\
\text { measles had a } \\
\text { progressive decline over } \\
\text { a three-year period } \\
\text {-Stakeholders found the } \\
\text { surveillance system to } \\
\text { be useful and } \\
\text { acceptable }\end{array}$ & III, B \\
\hline
\end{tabular}




\begin{tabular}{|c|c|c|c|c|c|c|c|c|c|c|}
\hline & & & & & & & & & $\begin{array}{l}\text {-System was unstable } \\
\text { due to high dependence } \\
\text { on donor funding and } \\
\text { support }\end{array}$ & \\
\hline 29. & Ghana & 2011 & Cholera & $\begin{array}{l}\text { Adjei et } \\
\text { al.[47] }\end{array}$ & 2017 & $\begin{array}{l}\text { To evaluate the } \\
\text { cholera } \\
\text { surveillance } \\
\text { system }\end{array}$ & $\begin{array}{l}\text { Evaluation study; } \\
\text { Records review; } \\
\text { Interviews }\end{array}$ & $\begin{array}{l}\text { Core functions: Case } \\
\text { registration, data } \\
\text { analysis, feedback } \\
\text { Support functions: } \\
\text { Resources } \\
\text { Attributes: Simplicity, } \\
\text { acceptability, stability, } \\
\text { flexibility, usefulness, } \\
\text { predictive value } \\
\text { positive, sensitivity, } \\
\text { timeliness, } \\
\text { representativeness }\end{array}$ & $\begin{array}{l}\text {-Case forms captured } \\
\text { sufficient information } \\
\text {-Data analysis was } \\
\text { insufficient and } \\
\text { incomplete } \\
\text {-Feedback was } \\
\text { encouraged across all } \\
\text { surveillance levels } \\
\text {-Adequate funding } \\
\text { support from } \\
\text { government and other } \\
\text { partners } \\
\text {-System was simple, } \\
\text { participants were } \\
\text { willing to participate in } \\
\text { the surveillance system, } \\
\text { it was flexible since it } \\
\text { adapted to changing } \\
\text { information needs or } \\
\text { operating conditions }\end{array}$ & III, B \\
\hline 30. & Nigeria & 2013 & $\begin{array}{l}\text { Cholera, } \\
\text { shigella, } \\
\text { measles, } \\
\text { tuberculosis, } \\
\text { hemorrhagic } \\
\text { diseases, } \\
\text { yellow fever, } \\
\text { human } \\
\text { influenza }\end{array}$ & $\begin{array}{l}\text { Jinadu et } \\
\text { al.[48] }\end{array}$ & 2018 & $\begin{array}{l}\text { To determine } \\
\text { the awareness } \\
\text { and knowledge } \\
\text { of health care } \\
\text { workers about } \\
\text { IDSR strategy } \\
\text { for epidemic } \\
\text { prone diseases }\end{array}$ & $\begin{array}{l}\text { Cross-sectional } \\
\text { facility-based study; } \\
\text { Cluster sampling; } \\
\text { Sample size (528); } \\
\text { Interviews }\end{array}$ & $\begin{array}{l}\text { Core functions: Case } \\
\text { registration, reporting, } \\
\text { Support functions: } \\
\text { Training, resources } \\
\text { Attributes: Simplicity }\end{array}$ & $\begin{array}{l}\text {-Reporting of epidemic } \\
\text { prone diseases was } \\
\text { time consuming } \\
\text {-Poor funding, } \\
\text { inadequate training and } \\
\text { retraining of health } \\
\text { workers, limited human } \\
\text { resource capacity and } \\
\text { lack of logistical support } \\
\text {-Reporting forms were } \\
\text { simple to complete }\end{array}$ & III, A \\
\hline
\end{tabular}

(a)Evidence Levels: Level I (Experimental studies, Randomized Controlled Trials); Level II (Quasi-experimental studies); Level III (Non-experimental studies)

${ }^{(b)}$ Quality Grades: A (High quality); B (Good Quality); C (Low Quality or major flaws) 


\section{Table 2. Summary of key study recommendations from the reviewed studies}

\begin{tabular}{|c|c|c|c|c|c|}
\hline $\begin{array}{l}\text { Surveillance } \\
\text { functions }\end{array}$ & Authors & $\begin{array}{l}\text { Publication } \\
\text { year }\end{array}$ & Country & Article title & Key study recommendations \\
\hline \multicolumn{6}{|c|}{ Recommendations on core functions } \\
\hline \multirow[t]{4}{*}{ Case confirmation } & Ngwa et al.[37] & 2016 & Cameroon & $\begin{array}{l}\text { Cholera public health } \\
\text { surveillance in the Republic of } \\
\text { Cameroon-opportunities and } \\
\text { challenges }\end{array}$ & $\begin{array}{l}\text { Equipping health facilities and districts with } \\
\text { surveillance personnel, computers and } \\
\text { laboratories. } \\
\text { Equipping health facility laboratories to } \\
\text { ensure early detection, confirmation and } \\
\text { rapid response. }\end{array}$ \\
\hline & Baghdadi[39] & 2016 & Sudan & $\begin{array}{l}\text { Assessment of core and } \\
\text { support functions of case- } \\
\text { based surveillance of } \\
\text { meningitis in hospitals in } \\
\text { Khartoum State in } 2015\end{array}$ & $\begin{array}{l}\text { Formulating and distributing protocols on } \\
\text { specimen (i.e. CSF) handling. }\end{array}$ \\
\hline & $\begin{array}{l}\text { Adokiya and } \\
\text { Awoonor- } \\
\text { Williams[40] }\end{array}$ & 2016 & Ghana & $\begin{array}{l}\text { Ebola virus disease surveillance } \\
\text { and response preparedness in } \\
\text { Northern Ghana }\end{array}$ & $\begin{array}{l}\text { Improving laboratory capacity and prompt } \\
\text { specimen taking. }\end{array}$ \\
\hline & Dairo et al.[19] & 2018 & Nigeria & $\begin{array}{l}\text { Compliance with epidemic- } \\
\text { prone diseases surveillance } \\
\text { and response guidelines among } \\
\text { health officers at surveillance } \\
\text { units in South-West Nigeria }\end{array}$ & $\begin{array}{l}\text { Strengthening laboratory support for disease } \\
\text { surveillance at the health facility level. }\end{array}$ \\
\hline \multirow[t]{3}{*}{ Reporting } & Nnebue et al.[27] & 2013 & Nigeria & $\begin{array}{l}\text { Effectiveness of data collection } \\
\text { and information transmission } \\
\text { process for disease notification } \\
\text { in Anambra State, Nigeria }\end{array}$ & $\begin{array}{l}\text { Provision of reporting forms and other } \\
\text { logistics on regular basis. } \\
\text { Expanding sources of reporting. }\end{array}$ \\
\hline & Lar et al.[24] & 2015 & Nigeria & $\begin{array}{l}\text { Challenges of integrated } \\
\text { disease surveillance response } \\
\text { reporting among healthcare } \\
\text { personnel in Mangu, Plateau } \\
\text { State, Nigeria }\end{array}$ & $\begin{array}{l}\text { Ensuring constant availability of IDSR } \\
\text { reporting forms in the health facilities. }\end{array}$ \\
\hline & $\begin{array}{l}\text { Adokiya et } \\
\text { al.(a)[21] }\end{array}$ & 2015 & Ghana & $\begin{array}{l}\text { Evaluation of the integrated } \\
\text { disease surveillance and } \\
\text { response system for infectious } \\
\text { diseases control in northern } \\
\text { Ghana }\end{array}$ & $\begin{array}{l}\text { Addressing inconsistences between weekly } \\
\text { and monthly surveillance data. }\end{array}$ \\
\hline
\end{tabular}




\begin{tabular}{|c|c|c|c|c|c|}
\hline & $\begin{array}{l}\text { Mwatondo et } \\
\text { al.[38] }\end{array}$ & 2016 & Kenya & $\begin{array}{l}\text { Factors associated with } \\
\text { adequate weekly reporting for } \\
\text { disease surveillance data } \\
\text { among health facilities in } \\
\text { Nairobi County, Kenya, } 2013\end{array}$ & $\begin{array}{l}\text { Providing urban settings with weekly } \\
\text { reporting tools. }\end{array}$ \\
\hline & Iwu et al.[25] & 2016 & Nigeria & $\begin{array}{l}\text { Assessment of disease } \\
\text { reporting among health care } \\
\text { workers in a South Eastern } \\
\text { State, Nigeria }\end{array}$ & $\begin{array}{l}\text { Improved coordination, communication and } \\
\text { support for disease reporting at local and } \\
\text { state levels. }\end{array}$ \\
\hline & Lakew et al.[43] & 2017 & Ethiopia & $\begin{array}{l}\text { Status of surveillance and } \\
\text { routine immunization } \\
\text { performances in Amhara } \\
\text { Region, Ethiopia: findings from } \\
\text { in-depth peer review }\end{array}$ & $\begin{array}{l}\text { Improvement of surveillance documentation } \\
\text { (i.e. copies of surveillance reports). }\end{array}$ \\
\hline & Ameh et al.[31] & 2015 & Nigeria & $\begin{array}{l}\text { Evaluation of the measles } \\
\text { surveillance system in Kaduna } \\
\text { State, Nigeria (2010-2012 }\end{array}$ & $\begin{array}{l}\text { Encourage all health facilities to be involved in } \\
\text { reporting }\end{array}$ \\
\hline & Jinadu et al.[48] & 2018 & Nigeria & $\begin{array}{l}\text { Integrated disease surveillance } \\
\text { and response strategy for } \\
\text { epidemic prone diseases at the } \\
\text { primary health care (PHC) level } \\
\text { in Oyo State, Nigeria: what do } \\
\text { health care workers know and } \\
\text { feel? }\end{array}$ & $\begin{array}{l}\text { Set up a good reward system to increase } \\
\text { willingness for reporting }\end{array}$ \\
\hline Feedback & Nnebue et al.[27] & 2013 & Nigeria & $\begin{array}{l}\text { Effectiveness of data collection } \\
\text { and information transmission } \\
\text { process for disease notification } \\
\text { in Anambra State, Nigeria }\end{array}$ & Ensuring adequate feedback of information. \\
\hline & Abubakar et al.[32] & 2013 & Nigeria & $\begin{array}{l}\text { Assessment of integrated } \\
\text { disease surveillance and } \\
\text { response strategy } \\
\text { implementation in selected } \\
\text { Local Government Areas of } \\
\text { Kaduna State }\end{array}$ & $\begin{array}{l}\text { Increased feedback from higher to lower } \\
\text { levels. }\end{array}$ \\
\hline & Nnebue et al.[26] & 2014 & Nigeria & $\begin{array}{l}\text { Challenges of disease } \\
\text { surveillance and notification in } \\
\text { Anambra State, Nigeria }\end{array}$ & $\begin{array}{l}\text { Promptly providing feedback within the } \\
\text { disease surveillance and notification system. }\end{array}$ \\
\hline
\end{tabular}




\begin{tabular}{|c|c|c|c|c|c|}
\hline & $\begin{array}{l}\text { Begashaw and } \\
\text { Tesfaye[28] }\end{array}$ & 2016 & Ethiopia & $\begin{array}{l}\text { Assessment of integrated } \\
\text { disease surveillance and } \\
\text { response implementation in } \\
\text { special health facilities of } \\
\text { Dawuro Zone }\end{array}$ & $\begin{array}{l}\text { Improved feedback from higher to lower } \\
\text { levels. }\end{array}$ \\
\hline & Benson et al.[20] & 2016 & South Africa & $\begin{array}{l}\text { Survey of the perceptions of } \\
\text { key stakeholders on the } \\
\text { attributes of the South African } \\
\text { Notifiable Diseases } \\
\text { Surveillance System }\end{array}$ & $\begin{array}{l}\text { Provision of feedback to all key stakeholders } \\
\text { in the Notifiable Disease Surveillance System. }\end{array}$ \\
\hline & Mboera et al.[33] & 2017 & Tanzania & $\begin{array}{l}\text { Malaria surveillance and use of } \\
\text { evidence in planning and } \\
\text { decision making in Kilosa } \\
\text { District, Tanzania }\end{array}$ & $\begin{array}{l}\text { Providing feedback to motivate timely } \\
\text { submission of reports. }\end{array}$ \\
\hline \multirow[t]{3}{*}{ Data analysis } & Adokiya et al.[22] & 2016 & Ghana & $\begin{array}{l}\text { Evaluation of the reporting } \\
\text { completeness and timeliness of } \\
\text { the integrated disease } \\
\text { surveillance and response } \\
\text { system in northern Ghana }\end{array}$ & $\begin{array}{l}\text { Initiation of plans to scale up data entry in } \\
\text { district health information management } \\
\text { systems at the periphery level to ensure data } \\
\text { accuracy. }\end{array}$ \\
\hline & Mboera et al.[33] & 2017 & Tanzania & $\begin{array}{l}\text { Malaria surveillance and use of } \\
\text { evidence in planning and } \\
\text { decision making in Kilosa } \\
\text { District, Tanzania }\end{array}$ & $\begin{array}{l}\text { Capacity strengthening on data analysis and } \\
\text { utilization at the facility and district levels. }\end{array}$ \\
\hline & Lakew et al.[43] & 2017 & Ethiopia & $\begin{array}{l}\text { Status of surveillance and } \\
\text { routine immunization } \\
\text { performances in Amhara } \\
\text { Region, Ethiopia: findings from } \\
\text { in-depth peer review }\end{array}$ & $\begin{array}{l}\text { Data analysis and surveillance performance } \\
\text { indicators monitoring at the zonal and district } \\
\text { (woreda) levels. }\end{array}$ \\
\hline \multicolumn{6}{|c|}{ Recommendations on support functions } \\
\hline \multirow[t]{2}{*}{ Training } & Nnebue et al.[27] & 2013 & Nigeria & $\begin{array}{l}\text { Effectiveness of data collection } \\
\text { and information transmission } \\
\text { process for disease notification } \\
\text { in Anambra State, Nigeria }\end{array}$ & $\begin{array}{l}\text { Periodic training and retraining of health } \\
\text { personnel on disease surveillance and } \\
\text { notification } \\
\text { Regular in-house training of health workers. }\end{array}$ \\
\hline & Nnebue et al.[26] & 2014 & Nigeria & $\begin{array}{l}\text { Challenges of disease } \\
\text { surveillance and notification in } \\
\text { Anambra State, Nigeria }\end{array}$ & $\begin{array}{l}\text { Increased training for health workers on } \\
\text { disease surveillance and notification. }\end{array}$ \\
\hline
\end{tabular}




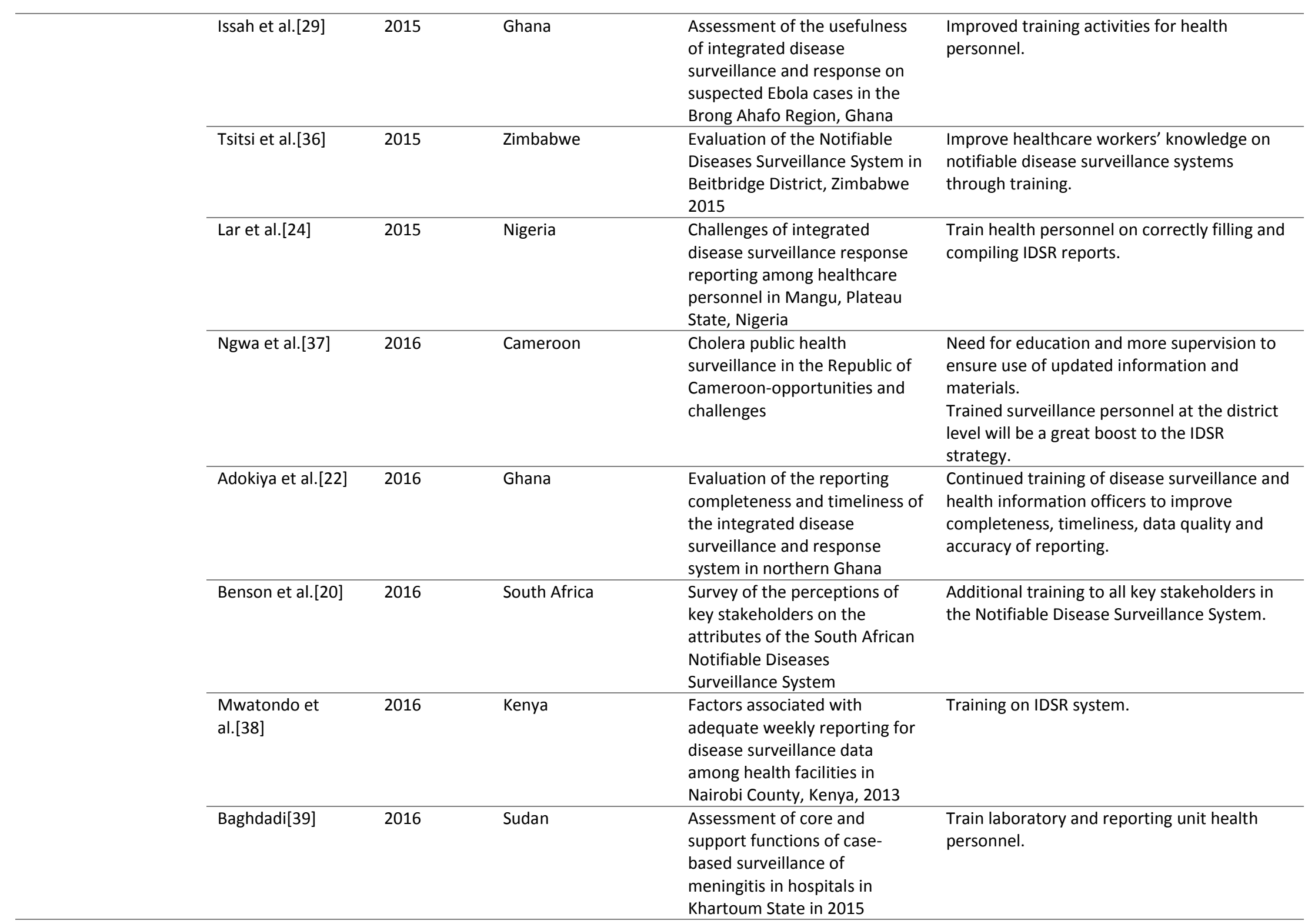




\begin{tabular}{|c|c|c|c|c|}
\hline Mairosi et al.[41] & 2016 & Zimbabwe & $\begin{array}{l}\text { Evaluation of notifiable disease } \\
\text { surveillance system in } \\
\text { Centenary District, Zimbabwe, } \\
2016\end{array}$ & $\begin{array}{l}\text { Health workers to be trained on IDSR } \\
\text { processes and the follow up actions. } \\
\text { Simplify training material to ease } \\
\text { understanding and improve knowledge levels. }\end{array}$ \\
\hline $\begin{array}{l}\text { Haakonde et } \\
\text { al.[23] }\end{array}$ & 2018 & Zambia & $\begin{array}{l}\text { Assessment of factors affecting } \\
\text { the implementation of the } \\
\text { integrated disease surveillance } \\
\text { and response in public health } \\
\text { care facilities: the case of } \\
\text { Rufunsa District, Zambia }\end{array}$ & $\begin{array}{l}\text { Ensure resources are secured and made } \\
\text { available towards the provision of regular } \\
\text { IDSR trainings targeting health care workers } \\
\text { engaged in IDSR implementation. }\end{array}$ \\
\hline Masiira et al.[46] & 2019 & Uganda & $\begin{array}{l}\text { Evaluation of integrated } \\
\text { disease surveillance and } \\
\text { response (IDSR) core and } \\
\text { support functions after the } \\
\text { revitalization of IDSR in Uganda } \\
\text { from } 2012 \text { to } 2016\end{array}$ & $\begin{array}{l}\text { Training of more health workers. } \\
\text { Regular IDSR training. } \\
\text { Incorporating IDSR training into the pre- } \\
\text { service curriculum for health workers. } \\
\text { Training of community members in IDSR. }\end{array}$ \\
\hline
\end{tabular}


health care workers know and

feel?

\begin{tabular}{|c|c|c|c|c|c|}
\hline \multirow[t]{7}{*}{ Supervision } & Nnebue et al.[27] & 2013 & Nigeria & $\begin{array}{l}\text { Effectiveness of data collection } \\
\text { and information transmission } \\
\text { process for disease notification } \\
\text { in Anambra State, Nigeria }\end{array}$ & $\begin{array}{l}\text { Improved supervision for surveillance data } \\
\text { collection and transmission. }\end{array}$ \\
\hline & Nnebue et al.[26] & 2014 & Nigeria & $\begin{array}{l}\text { Challenges of disease } \\
\text { surveillance and notification in } \\
\text { Anambra State, Nigeria }\end{array}$ & Ensuring adequate supervision. \\
\hline & Tsitsi et al.[36] & 2015 & Zimbabwe & $\begin{array}{l}\text { Evaluation of the Notifiable } \\
\text { Diseases Surveillance System in } \\
\text { Beitbridge District, Zimbabwe } \\
2015\end{array}$ & $\begin{array}{l}\text { Support and supervision to ensure notifiable } \\
\text { diseases are notified using the correct } \\
\text { channels. }\end{array}$ \\
\hline & Ngwa et al.[37] & 2016 & Cameroon & $\begin{array}{l}\text { Cholera public health } \\
\text { surveillance in the Republic of } \\
\text { Cameroon-opportunities and } \\
\text { challenges }\end{array}$ & $\begin{array}{l}\text { Increased supervision to ensure use of } \\
\text { updated information and materials. }\end{array}$ \\
\hline & Lakew et al.[43] & 2017 & Ethiopia & $\begin{array}{l}\text { Status of surveillance and } \\
\text { routine immunization } \\
\text { performances in Amhara } \\
\text { Region, Ethiopia: findings from } \\
\text { in-depth peer review }\end{array}$ & $\begin{array}{l}\text { Ensuring strict adherence to planned } \\
\text { surveillance schedules (i.e. supervisory visits) }\end{array}$ \\
\hline & $\begin{array}{l}\text { Haakonde et } \\
\text { al.[23] }\end{array}$ & 2018 & Zambia & $\begin{array}{l}\text { Assessment of factors affecting } \\
\text { the implementation of the } \\
\text { integrated disease surveillance } \\
\text { and response in public health } \\
\text { care facilities: the case of } \\
\text { Rufunsa District, Zambia }\end{array}$ & $\begin{array}{l}\text { Ensuring mentorship, regular and scheduled } \\
\text { supervision is provided to strengthen IDSR } \\
\text { implementation at the district level. }\end{array}$ \\
\hline & Masiira et al.[46] & 2019 & Uganda & $\begin{array}{l}\text { Evaluation of integrated } \\
\text { disease surveillance and } \\
\text { response (IDSR) core and } \\
\text { support functions after the } \\
\text { revitalization of IDSR in Uganda } \\
\text { from } 2012 \text { to } 2016\end{array}$ & $\begin{array}{l}\text { Strengthening supervision to improve IDSR } \\
\text { performance. }\end{array}$ \\
\hline Resources & Abubakar et al.[32] & 2013 & Nigeria & $\begin{array}{l}\text { Assessment of integrated } \\
\text { disease surveillance and } \\
\text { response strategy } \\
\text { implementation in selected } \\
\text { Local Government Areas of } \\
\text { Kaduna State }\end{array}$ & $\begin{array}{l}\text { Provision of sufficient logistical resources and } \\
\text { data management tools. }\end{array}$ \\
\hline
\end{tabular}




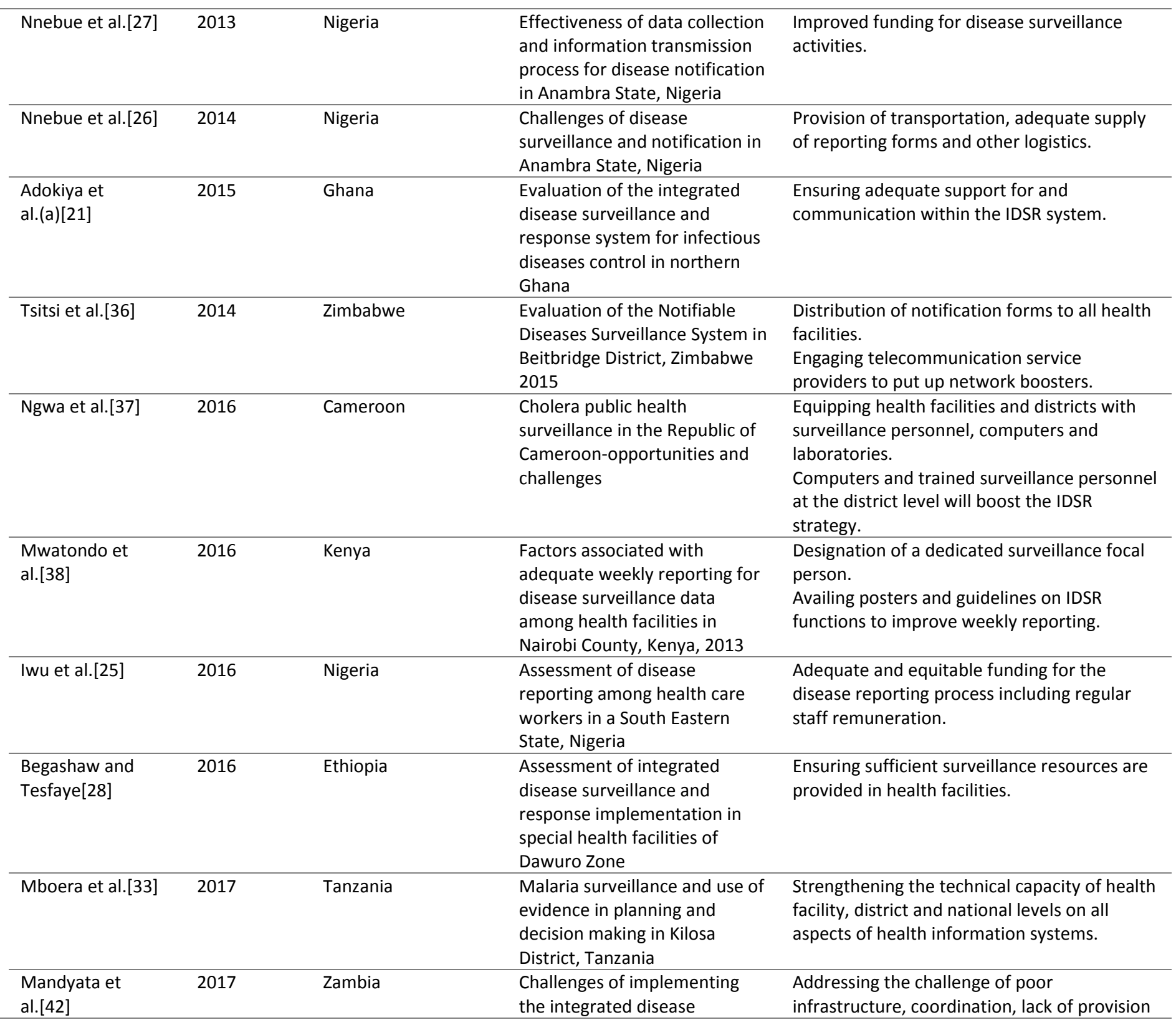




\begin{tabular}{|c|c|c|c|c|c|}
\hline & & & & $\begin{array}{l}\text { surveillance and response } \\
\text { strategy in Zambia: a health } \\
\text { worker perspective }\end{array}$ & $\begin{array}{l}\text { of optimal technical support to DHMTs and } \\
\text { health facilities. }\end{array}$ \\
\hline & Dairo et al.[19] & 2018 & Nigeria & $\begin{array}{l}\text { Compliance with epidemic- } \\
\text { prone diseases surveillance } \\
\text { and response guidelines among } \\
\text { health officers at surveillance } \\
\text { units in South-West Nigeria }\end{array}$ & $\begin{array}{l}\text { Ensuring funds provision and other resources } \\
\text { to surveillance workers to achieve effective } \\
\text { disease control. }\end{array}$ \\
\hline & $\begin{array}{l}\text { Haakonde et } \\
\text { al.[23] }\end{array}$ & 2018 & Zambia & $\begin{array}{l}\text { Assessment of factors affecting } \\
\text { the implementation of the } \\
\text { integrated disease surveillance } \\
\text { and response in public health } \\
\text { care facilities: the case of } \\
\text { Rufunsa District, Zambia }\end{array}$ & $\begin{array}{l}\text { Allocating funds to support IDSR activities in } \\
\text { the health sector budget. }\end{array}$ \\
\hline & Masiira et al.[46] & 2019 & Uganda & $\begin{array}{l}\text { Evaluation of integrated } \\
\text { disease surveillance and } \\
\text { response (IDSR) core and } \\
\text { support functions after the } \\
\text { revitalization of IDSR in Uganda } \\
\text { from } 2012 \text { to } 2016\end{array}$ & $\begin{array}{l}\text { Increasing IDSR funding at district and health } \\
\text { facility levels. }\end{array}$ \\
\hline & Ameh et al.[31] & 2016 & Nigeria & $\begin{array}{l}\text { Evaluation of the measles } \\
\text { surveillance system in Kaduna } \\
\text { State, Nigeria (2010-2012) }\end{array}$ & $\begin{array}{l}\text { Sustained provision for funding and increased } \\
\text { logistical support }\end{array}$ \\
\hline Recommendatic & surveillance attrib & tes & & & \\
\hline $\begin{array}{l}\text { Timeliness and } \\
\text { completeness }\end{array}$ & Nnebue et al.[27] & 2013 & Nigeria & $\begin{array}{l}\text { Effectiveness of data collection } \\
\text { and information transmission } \\
\text { process for disease notification } \\
\text { in Anambra State, Nigeria }\end{array}$ & $\begin{array}{l}\text { Increased awareness on importance of } \\
\text { effective reporting. }\end{array}$ \\
\hline & Maponga et al.[34] & 2014 & Zimbabwe & $\begin{array}{l}\text { Evaluation of the notifiable } \\
\text { diseases surveillance system in } \\
\text { Sanyati district, Zimbabwe, } \\
\text { 2010-2011 }\end{array}$ & $\begin{array}{l}\text { Availing information on when diseases are } \\
\text { being notified. }\end{array}$ \\
\hline & Issah et al.[29] & 2015 & Ghana & $\begin{array}{l}\text { Assessment of the usefulness } \\
\text { of integrated disease } \\
\text { surveillance and response on } \\
\text { suspected Ebola cases in the } \\
\text { Brong Ahafo Region, Ghana }\end{array}$ & $\begin{array}{l}\text { Improved timely reporting of notifiable } \\
\text { conditions. }\end{array}$ \\
\hline & Adokiya et al.[22] & 2016 & Ghana & $\begin{array}{l}\text { Evaluation of the reporting } \\
\text { completeness and timeliness of }\end{array}$ & $\begin{array}{l}\text { Consistency during weekly and monthly } \\
\text { reporting. }\end{array}$ \\
\hline
\end{tabular}




\begin{tabular}{|c|c|c|c|c|c|}
\hline & & & & $\begin{array}{l}\text { the integrated disease } \\
\text { surveillance and response } \\
\text { system in northern Ghana }\end{array}$ & $\begin{array}{l}\text { Conducting further investigations to address } \\
\text { reporting completeness. }\end{array}$ \\
\hline & Mairosi et al.[41] & 2016 & Zimbabwe & $\begin{array}{l}\text { Evaluation of notifiable disease } \\
\text { surveillance system in } \\
\text { Centenary District, Zimbabwe, } \\
2016\end{array}$ & $\begin{array}{l}\text { Provision of time information on disease } \\
\text { notification. }\end{array}$ \\
\hline & Benson et al.[20] & 2016 & South Africa & $\begin{array}{l}\text { Survey of the perceptions of } \\
\text { key stakeholders on the } \\
\text { attributes of the South African } \\
\text { Notifiable Diseases } \\
\text { Surveillance System }\end{array}$ & $\begin{array}{l}\text { Health reforms to address surveillance system } \\
\text { reporting timeliness. }\end{array}$ \\
\hline & Ngwa et al.[37] & 2016 & Cameroon & $\begin{array}{l}\text { Cholera public health } \\
\text { surveillance in the Republic of } \\
\text { Cameroon-opportunities and } \\
\text { challenges }\end{array}$ & Enhancing human resource capacity. \\
\hline & Mboera et al.[33] & 2017 & Tanzania & $\begin{array}{l}\text { Malaria surveillance and use of } \\
\text { evidence in planning and } \\
\text { decision making in Kilosa } \\
\text { District, Tanzania }\end{array}$ & Awareness on specific reporting dates. \\
\hline & $\begin{array}{l}\text { Randriamiarana et } \\
\text { al.[45] }\end{array}$ & 2018 & Madagascar & $\begin{array}{l}\text { Evaluation of the reinforced } \\
\text { integrated disease surveillance } \\
\text { and response strategy using } \\
\text { short message service data } \\
\text { transmission in two southern } \\
\text { regions of Madagascar, 2014- } \\
15\end{array}$ & $\begin{array}{l}\text { Reducing workload, increasing training and } \\
\text { improving mobile network infrastructure. }\end{array}$ \\
\hline & Masiira et al.[46] & 2019 & Uganda & $\begin{array}{l}\text { Evaluation of integrated } \\
\text { disease surveillance and } \\
\text { response (IDSR) core and } \\
\text { support functions after the } \\
\text { revitalization of IDSR in Uganda } \\
\text { from } 2012 \text { to } 2016\end{array}$ & $\begin{array}{l}\text { Enhanced IDSR training and adopting mobile- } \\
\text { based reporting. }\end{array}$ \\
\hline \multirow[t]{2}{*}{$\begin{array}{l}\text { Data } \\
\text { Quality/Accuracy }\end{array}$} & Maponga et al.[34] & 2014 & Zimbabwe & $\begin{array}{l}\text { Evaluation of the notifiable } \\
\text { diseases surveillance system in } \\
\text { Sanyati district, Zimbabwe, } \\
\text { 2010-2011 }\end{array}$ & Ensuring complete and precise reporting. \\
\hline & Mairosi et al.[41] & 2016 & Zimbabwe & $\begin{array}{l}\text { Evaluation of notifiable disease } \\
\text { surveillance system in } \\
\text { Centenary District, Zimbabwe, } \\
2016\end{array}$ & Reducing missed data occurrences. \\
\hline
\end{tabular}




\begin{tabular}{|c|c|c|c|c|c|}
\hline & Adokiya et al.[22] & 2016 & Ghana & $\begin{array}{l}\text { Evaluation of the reporting } \\
\text { completeness and timeliness of } \\
\text { the integrated disease } \\
\text { surveillance and response } \\
\text { system in northern Ghana }\end{array}$ & $\begin{array}{l}\text { Adopting the DHIS2 reporting system starting } \\
\text { from the peripheral level. }\end{array}$ \\
\hline & $\begin{array}{l}\text { Randriamiarana et } \\
\text { al.[45] }\end{array}$ & 2018 & Madagascar & $\begin{array}{l}\text { Evaluation of the reinforced } \\
\text { integrated disease surveillance } \\
\text { and response strategy using } \\
\text { short message service data } \\
\text { transmission in two southern } \\
\text { regions of Madagascar, 2014- } \\
15\end{array}$ & $\begin{array}{l}\text { Providing quality control mechanisms to avoid } \\
\text { transmission of erroneous data. }\end{array}$ \\
\hline \multirow[t]{2}{*}{ Usefulness } & Benson et al.[20] & 2016 & South Africa & $\begin{array}{l}\text { Survey of the perceptions of } \\
\text { key stakeholders on the } \\
\text { attributes of the South African } \\
\text { Notifiable Diseases } \\
\text { Surveillance System }\end{array}$ & $\begin{array}{l}\text { Health reforms to encourage use of } \\
\text { surveillance data. }\end{array}$ \\
\hline & Mairosi et al.[41] & 2016 & Zimbabwe & $\begin{array}{l}\text { Evaluation of notifiable disease } \\
\text { surveillance system in } \\
\text { Centenary District, Zimbabwe, } \\
2016\end{array}$ & $\begin{array}{l}\text { Documenting public health action reports } \\
\text { based on surveillance data. }\end{array}$ \\
\hline \multirow[t]{3}{*}{ Acceptability } & Maponga et al.[34] & 2014 & Zimbabwe & $\begin{array}{l}\text { Evaluation of the notifiable } \\
\text { diseases surveillance system in } \\
\text { Sanyati district, Zimbabwe, } \\
\text { 2010-2011 }\end{array}$ & Provision of clear job descriptions. \\
\hline & Tsitsi et al.[36] & 2015 & Zimbabwe & $\begin{array}{l}\text { Evaluation of the Notifiable } \\
\text { Diseases Surveillance System in } \\
\text { Beitbridge District, Zimbabwe } \\
2015\end{array}$ & $\begin{array}{l}\text { Aligning surveillance activities with day-to-day } \\
\text { duties. }\end{array}$ \\
\hline & Mairosi et al.[41] & 2016 & Zlmbabwe & $\begin{array}{l}\text { Evaluation of notifiable disease } \\
\text { surveillance system in } \\
\text { Centenary District, Zimbabwe, } \\
2016\end{array}$ & Designation of surveillance focal persons. \\
\hline \multirow[t]{2}{*}{ Stability } & Maponga et al.[34] & 2014 & Zimbabwe & $\begin{array}{l}\text { Evaluation of the notifiable } \\
\text { diseases surveillance system in } \\
\text { Sanyati district, Zimbabwe, } \\
2010-2011\end{array}$ & Availability of human resources. \\
\hline & Tsitsi et al.[36] & 2015 & Zimbabwe & $\begin{array}{l}\text { Evaluation of the Notifiable } \\
\text { Diseases Surveillance System in } \\
\text { Beitbridge District, Zimbabwe } \\
2015\end{array}$ & Availing material resources. \\
\hline
\end{tabular}




\begin{tabular}{|c|c|c|c|c|c|}
\hline & Mairosi et al.[41] & 2016 & Zimbabwe & $\begin{array}{l}\text { Evaluation of notifiable disease } \\
\text { surveillance system in } \\
\text { Centenary District, Zimbabwe, } \\
2016\end{array}$ & $\begin{array}{l}\text { Enhanced training and provision of } \\
\text { communication and logistical facilities. }\end{array}$ \\
\hline & $\begin{array}{l}\text { Mandyata et } \\
\text { al.[42] }\end{array}$ & 2017 & Zambia & $\begin{array}{l}\text { Challenges of implementing } \\
\text { the integrated disease } \\
\text { surveillance and response } \\
\text { strategy in Zambia: a health } \\
\text { worker perspective }\end{array}$ & $\begin{array}{l}\text { Improved internet connectivity and } \\
\text { infrastructure. }\end{array}$ \\
\hline & Benson et al.[30] & 2017 & South Africa & $\begin{array}{l}\text { Comparing laboratory } \\
\text { surveillance with the notifiable } \\
\text { diseases surveillance system in } \\
\text { South Africa }\end{array}$ & Provision of reliable diagnostic equipment. \\
\hline \multirow[t]{5}{*}{ Simplicity } & Maponga et al.[34] & 2014 & Zimbabwe & $\begin{array}{l}\text { Evaluation of the notifiable } \\
\text { diseases surveillance system in } \\
\text { Sanyati district, Zimbabwe, } \\
\text { 2010-2011 }\end{array}$ & Ease of completing notification forms. \\
\hline & Tsitsi et al.[36] & 2015 & Zimbabwe & $\begin{array}{l}\text { Evaluation of the notifiable } \\
\text { Diseases Surveillance System in } \\
\text { Beitbridge District, Zimbabwe } \\
2015\end{array}$ & $\begin{array}{l}\text { Reduced length of time required to complete } \\
\text { notification forms. }\end{array}$ \\
\hline & Mairosi et al.[41] & 2016 & Zimbabwe & $\begin{array}{l}\text { Evaluation of notifiable disease } \\
\text { surveillance system in } \\
\text { Centenary District, Zimbabwe, } \\
2016\end{array}$ & $\begin{array}{l}\text { Ease of understanding surveillance system } \\
\text { functionalities. }\end{array}$ \\
\hline & Benson et al.[20] & 2016 & South Africa & $\begin{array}{l}\text { Survey of the perceptions of } \\
\text { key stakeholders on the } \\
\text { attributes of the South African } \\
\text { Notifiable Diseases } \\
\text { Surveillance System }\end{array}$ & $\begin{array}{l}\text { Simplification of surveillance system at } \\
\text { operational level. }\end{array}$ \\
\hline & $\begin{array}{l}\text { Randriamiarana et } \\
\text { al.[45] }\end{array}$ & 2018 & Madagascar & $\begin{array}{l}\text { Evaluation of the reinforced } \\
\text { integrated disease surveillance } \\
\text { and response strategy using } \\
\text { short message service data } \\
\text { transmission in two southern } \\
\text { regions of Madagascar, 2014- } \\
15\end{array}$ & $\begin{array}{l}\text { Distribution and display of simplified and } \\
\text { understandable terms of reference and case } \\
\text { definition guidelines. }\end{array}$ \\
\hline \multicolumn{6}{|c|}{ Further recommendations } \\
\hline & Maponga et al.[34] & 2014 & Zimbabwe & $\begin{array}{l}\text { Evaluation of the notifiable } \\
\text { diseases surveillance system in }\end{array}$ & $\begin{array}{l}\text { Need for the Ministry of Health to develop an } \\
\text { electronic based system for surveillance data }\end{array}$ \\
\hline
\end{tabular}




\begin{tabular}{|c|c|c|c|c|c|}
\hline \multirow{8}{*}{$\begin{array}{l}\text { Alternative } \\
\text { surveillance } \\
\text { strategies }\end{array}$} & & & & $\begin{array}{l}\text { Sanyati district, Zimbabwe, } \\
\text { 2010-2011 }\end{array}$ & $\begin{array}{l}\text { reporting riding on the availability of mobile } \\
\text { phone use. }\end{array}$ \\
\hline & Tsitsi et al.[36] & 2015 & Zimbabwe & $\begin{array}{l}\text { Evaluation of the notifiable } \\
\text { Diseases Surveillance System in } \\
\text { Beitbridge District, Zimbabwe } \\
2015\end{array}$ & $\begin{array}{l}\text { Adoption of an electronic/mobile channel in } \\
\text { notifying diseases to cut down on costs of the } \\
\text { paper-based system. }\end{array}$ \\
\hline & $\begin{array}{l}\text { Issah et } \\
\text { al.[29] }\end{array}$ & 2015 & Ghana & $\begin{array}{l}\text { Assessment of the usefulness } \\
\text { of integrated disease } \\
\text { surveillance and response on } \\
\text { suspected Ebola cases in the } \\
\text { Brong Ahafo Region, Ghana }\end{array}$ & $\begin{array}{l}\text { Improving and focusing on community based } \\
\text { surveillance system by bringing it into the } \\
\text { mainstream surveillance for Ebola Viral } \\
\text { Disease. }\end{array}$ \\
\hline & Ngwa et al.[37] & 2016 & Cameroon & $\begin{array}{l}\text { Cholera public health } \\
\text { surveillance in the Republic of } \\
\text { Cameroon-opportunities and } \\
\text { challenges }\end{array}$ & $\begin{array}{l}\text { Equipping all health facilities with the 'green } \\
\text { line' mobile surveillance approach. }\end{array}$ \\
\hline & Benson et al.[20] & 2016 & South Africa & $\begin{array}{l}\text { Survey of the perceptions of } \\
\text { key stakeholders on the } \\
\text { attributes of the South African } \\
\text { Notifiable Diseases } \\
\text { Surveillance System }\end{array}$ & $\begin{array}{l}\text { Introducing an electronic system including use } \\
\text { of mobile telephone technology to address } \\
\text { perceived weaknesses of the NDSS. }\end{array}$ \\
\hline & Lakew et al.[43] & 2017 & Ethiopia & $\begin{array}{l}\text { Status of surveillance and } \\
\text { routine immunization } \\
\text { performances in Amhara } \\
\text { Region, Ethiopia: findings from } \\
\text { in-depth peer review }\end{array}$ & $\begin{array}{l}\text { Properly formulating operational plans to } \\
\text { improve active case search with realistic } \\
\text { prioritization of visiting reporting sites. }\end{array}$ \\
\hline & Wu et al.[44] & 2018 & Malawi & $\begin{array}{l}\text { Integrated Disease Surveillance } \\
\text { and Response (IDSR) in Malawi: } \\
\text { Implementation gaps and } \\
\text { challenges for timely alert }\end{array}$ & $\begin{array}{l}\text { Improved technology infrastructure and } \\
\text { adapting mobile technologies. } \\
\text { Utilization of syndromic surveillance approach } \\
\text { combined with systematic virological testing. }\end{array}$ \\
\hline & $\begin{array}{l}\text { Randriamiarana et } \\
\text { al.[45] }\end{array}$ & 2018 & Madagascar & $\begin{array}{l}\text { Evaluation of the reinforced } \\
\text { integrated disease surveillance } \\
\text { and response strategy using } \\
\text { short message service data } \\
\text { transmission in two southern } \\
\text { regions of Madagascar, 2014- } \\
15\end{array}$ & $\begin{array}{l}\text { Improved data collection, compilation and } \\
\text { transfer through an electronic system. } \\
\text { Increasing mobile network coverage. }\end{array}$ \\
\hline Further research & Maponga et al.[34] & 2014 & Zimbabwe & $\begin{array}{l}\text { Evaluation of the notifiable } \\
\text { diseases surveillance system in } \\
\text { Sanyati district, Zimbabwe, } \\
2010-2011\end{array}$ & $\begin{array}{l}\text { Need for further research on the effect of } \\
\text { training health-care workers on the } \\
\text { surveillance system. }\end{array}$ \\
\hline
\end{tabular}




\begin{tabular}{|c|c|c|c|c|c|}
\hline & Adokiya et al.[22] & 2016 & Ghana & $\begin{array}{l}\text { Evaluation of the reporting } \\
\text { completeness and timeliness of } \\
\text { the integrated disease } \\
\text { surveillance and response } \\
\text { system in northern Ghana }\end{array}$ & $\begin{array}{l}\text { Further research to improve reporting } \\
\text { completeness and timeliness of surveillance } \\
\text { data. }\end{array}$ \\
\hline & $\begin{array}{l}\text { Mwatondo et } \\
\text { al.[38] }\end{array}$ & 2016 & Kenya & $\begin{array}{l}\text { Factors associated with } \\
\text { adequate weekly reporting for } \\
\text { disease surveillance data } \\
\text { among health facilities in } \\
\text { Nairobi County, Kenya, } 2013\end{array}$ & $\begin{array}{l}\text { Conducting further studies in rural or mixed } \\
\text { settings in different Kenyan counties in order } \\
\text { to gather information on the challenges of } \\
\text { reporting in health facilities. }\end{array}$ \\
\hline $\begin{array}{l}\text { Other } \\
\text { recommendations }\end{array}$ & $\begin{array}{l}\text { Adokiya et al. } \\
\text { 2015(b)[35] }\end{array}$ & 2015 & Ghana & $\begin{array}{l}\text { The integrated disease } \\
\text { surveillance and response } \\
\text { system in Northern Ghana: } \\
\text { challenges to the core and } \\
\text { support functions }\end{array}$ & $\begin{array}{l}\text { Need to recognize disease surveillance } \\
\text { activities as essential for the overall } \\
\text { functioning of the health system. }\end{array}$ \\
\hline
\end{tabular}




\section{Core functions}

\section{Case Confirmation}

Four of the 30 reviewed studies recommended strengthened case confirmation capacities [19, 37, 39, 40]. Of these, two studies reported that prompt public health action requires enhanced laboratory capacity $[19,37]$. Laboratory services absence in health facilities to confirm cholera cases and outbreaks was reported in Cameroon [37]. Therefore, this required laboratory facilities and equipment be provided to ensure prompt detection, confirmation and response to cholera cases [37]. Similarly, laboratory capacity strengthening and prompt specimen collection was recommended in Ghana to ensure adequate surveillance and response preparedness to Ebola [40]. A sub-theme based on a recommendation derived from the reviewed studies alluded to improved specimen handling [39]. Functions relating to case confirmation were absent in health facilities in Khartoum State, hence necessitating need to formulate and distribute protocols for specimen handling specific to meningitis [39].

\section{Reporting}

Slightly more than a quarter $(8 / 30)$ of the reviewed studies provided recommendations on improving surveillance reporting $[21,24,25,27,31,38,43,48]$. Of these studies, two main subthemes were identified on improved reporting quality $[21,25,31,48]$ and adequate provision of reporting forms $[24,27,38,43]$. Health workers' awareness on the link between their day-to-day activities and disease reporting will improve their willingness to adhere to reporting guidelines [25]. A study in Kenya reported having weekly reporting forms present in health facilities significantly increased disease surveillance reporting odds [38]. Therefore, availing IDSR reporting tools would ensure continuity and consistency in reporting surveillance data [24, 38]. In Ghana, inaccuracies and missing data in surveillance reports submitted from peripheral to regional 
level resulted from uncertainties on the most appropriate reporting channel [21]. This required addressing inconsistencies of weekly and monthly reports submitted through the various channels [21]. Advocating for improvements and clarity on the proper reporting channels would avoid frequent communication breakdowns and missing data in surveillance reports [21]. Improved surveillance documentation was recommended since most regional surveillance offices lacked active case searches written reports from reporting sites in Ethiopia [43]. An efficient reward system for reporting would motivate health personnel reporting efforts and involvement in the surveillance system [48].

\section{Feedback}

Up to $20 \%$ (6/30) of the studies recommended the need for improved feedback [20, 26-28, 32, 33]. Further, two key sub-themes emanated from the reviewed studies on improving feedback, which identified the need for improved health workers' attitudes [20] and enhanced feedback from the higher to lower levels $[28,32,33]$. Feedback on reported data influences health worker's attitudes and willingness to participate in surveillance activities. However, inadequate feedback to health facilities may demotivate health workers, limiting their efforts towards efficient and timely reporting [33]. Health worker's low perceptions on the disease surveillance system's acceptability, flexibility and usefulness would be resolved through sufficient feedback [20]. Adequate feedback provision to motivate health workers to submit timely reports for malaria cases would address inefficient disease surveillance reporting in Tanzania [33]. Similarly, improved feedback from higher to lower levels would motivate health staff to report efficiently and influence their performance in surveillance activities as reported in Nigeria and Ethiopia [28, 32]. Furthermore, ensuring adequate and prompt feedback within disease surveillance and notification system would alleviate major challenges faced within the system [26, 27]. 


\section{Data Analysis}

Of the studies (3/30) recommending for increased data analysis, one study indicated that data transmission challenges using paper-based reporting from periphery to district level increased error introduction likelihood in the reported data [22]. Hence, necessitating plans initiation for scaling up data entry in DHIMS2 at the periphery level to improve data accuracy in Ghana [22]. Similarly, challenges involving limited capacity and low evidence of proper data analysis at the hospital and district levels in Tanzania were to be mitigated by strengthening capacity for data analysis and availing tallying sheets, register books and reporting forms [33]. Further, limited use of outcomes from surveillance performance analysis in Ethiopia required an undertaking to analyse surveillance data and closely monitor surveillance performance indicators at regional levels [43]. The subthemes derived from studies recommending routine data analysis were centered on surveillance system performance monitoring [43] and improved data accuracy [22, 33].

\section{Support functions}

\section{Training}

Sixty percent (18/30) of studies in the review recommended for enhanced training of health personnel. Three major sub-themes were derived from study recommendations regarding surveillance training and this included improved surveillance system performance [23, 34, 37], improved surveillance data quality $[21,24,25,39,42]$ and enhanced knowledge on surveillance systems [26, 27, 29, 36, 38, 41, 45-48]. Low knowledge on correct forms for reporting notifiable diseases negatively affected timely disease reporting in Zimbabwe [36]. Therefore, health workers required training to improve their knowledge on notifiable disease surveillance systems through induction and on job training [36]. On-job training of health personnel during supervisory visits 
and sensitisation meetings is the common strategy applied or recommended especially at health facility level with limited formal training on IDSR implementation [29, 34, 36]. On the other hand, improved reporting practices as a result of forms availability and recognising health workers' reporting efforts was significantly associated with a post-basic training intervention in Nigeria [24]. Consequently, continued health worker training on correct form filling and reports compilation was recommended [24]. In Ghana, formal IDSR training with a focus on detection and reporting of Ebola Viral Disease (EVD) suspected cases was lacking at health facility and community levels [29]. Hence, an integrated and sustained funding support towards health personnel training at facility and community levels would ensure effective EVD suspected cases contact tracing and halt disease transmission [29]. Disease surveillance training especially at community, heath facility and district levels was limited in comparison to training undertaken at the regional and national levels in Cameroon [37]. The mitigation measure recommended was to increase health personnel training at district level to enhance IDSR strategy implementation [37]. Previous studies conducted in West Africa recommended regular training of health staff to improve reporting and mitigate other challenges associated with inadequate training [25-27, 47, 48]. Furthermore, IDSR training was inadequate in Zambia resulting in health worker dependence on prior knowledge while executing their duties [42]. Therefore, they required adequate training to improve the quality and quantity of surveillance data being generated and utilised for decisionmaking [42]. Health workers' training needs on IDSR system aspects needed addressing to enable proper identification of designated focal surveillance persons in Kenya [38]. In South Sudan, increased health personnel training in hospital reporting units and laboratories would improve meningitis case-based reporting within the surveillance system [39]. Although heath workers in Zimbabwe perceived notifiable disease surveillance system to be simple and easy to use, training 
medRxiv preprint doi: https://doi.org/10.1101/2020.09.08.20190553; this version posted September 9, 2020. The copyright holder for this preprint (which was not certified by peer review) is the author/funder, who has granted medRxiv a license to display the preprint in perpetuity.

It is made available under a CC-BY-NC-ND 4.0 International license .

was necessary to increase their understanding of IDSR processes and follow up actions. This would be achieved through simplifying training materials to ease understanding of the system [41]. In Zambia, most respondents felt securing adequate funds to conduct periodical training and retraining could strengthen all IDSR system implementation aspects [23]. Similarly, challenges associated with inadequate training in Nigeria was to be mitigated through regular in-house training and re-training of health personnel on disease surveillance and notification [26, 27]. Further, in Uganda, having an inadequate number of frontline health personnel trained on IDSR system was to be resolved through IDSR training incorporation in health worker's initial preservice curriculum and community involvement in training [46].

\section{Supervision}

Seven out of the 30 studies indicated the need for supervision of surveillance activities [23, 26, 27 , 36, 37, 43, 46]. The sub-themes relating to supervision that emanated from the study recommendations were based on strengthening implementation of the surveillance system [23, 27, 43, 46], utilisation of up-to-date information [37] and identification of correct reporting channels $[27,36]$. Enhanced surveillance supervisory efforts at health facility level would ensure notifiable diseases are notified through correct channels [36]. However, most supervisory reviews only focused on surveillance activities involving immunisable diseases, tuberculosis and HIV/AIDs [36]. Similarly, partial supervision was undertaken in Cameroon at regional and district levels, while at community and health facility levels there was complete absence of supervisory activities [37]. Increased awareness on supervision benefits and efforts to enhance supervision would ensure utilisation of up-to-date surveillance information and materials amongst HCWs [37]. Surveillance focal persons irregularly provided supportive supervision for active case searches in Ethiopia, hence requiring strict adherence to planned surveillance schedules for conducting supervisory 
medRxiv preprint doi: https://doi.org/10.1101/2020.09.08.20190553; this version posted September 9, 2020. The copyright holder for this preprint (which was not certified by peer review) is the author/funder, who has granted medRxiv a license to display the preprint in perpetuity.

It is made available under a CC-BY-NC-ND 4.0 International license .

visits [43]. Furthermore, health workers at the district level in Zambia felt regular and scheduled supervisory assistance from higher levels would strengthen the IDSR system [23]. In addition, increased supervision was required to ensure disease notification systems were effective in data collection and information transmission in Nigeria [27]. Irregular supervision was still an existing challenge in the revitalised IDSR programme that required addressing to improve IDSR performance in Uganda [46].

\section{Resources}

Slightly more than half (16/30) of the studies identified the need for sufficient resources to support surveillance activities. Of the reviewed studies, recommendations on increased resource support for surveillance activities were focused on financial resources [23, 25, 27, 31, 46], human resources [37, 38], materials and logistical support $[19,26,28,31-33,36,38]$ and equipment and infrastructure $[21,36,37,42]$. Surveillance data analysis and management tools unavailability at health facility and district levels was reported in Cameroon [37]. Hence, requiring health facilities and district levels to be equipped with computers [37]. Similarly, data management tools availability was to be complemented by their functionality to ease surveillance data entry and analysis $[28,32]$. On the other hand, the main challenge facing cellphone communication reporting channels was poor network infrastructure in Zimbabwe [36]. Hence, requiring telecommunication service providers engagement to set up network boosters to improve communication and timely reporting [36]. Limited utilisation of routine health information for performance monitoring was to be mitigated through health information systems strengthening at all surveillance levels in Tanzania [33]. Notification forms unavailability in Zimbabwe hindered HCWs efforts for disease notification and delayed epidemic investigations [36]. Therefore, this necessitated the distribution of notification forms to all health facilities [36]. In Kenya, health facilities displaying visual aids 
for IDSR functions were more likely to report surveillance data [38]. Hence, to strengthen these efforts it was recommended that posters and guidelines on IDSR functions be provided to improve reporting [38]. In Zambia, IDSR technical guidelines were unavailable in health facilities, hence they lacked the appropriate procedures for handling suspected cases of notifiable diseases [42]. This identified the need for technical support especially at health facility levels to promote and improve early disease detection [42].

Health facilities lacking health workers designated to manage disease surveillance data had decreased odds of adequate reporting [38]. Hence, designating a surveillance focal person in health facilities would improve surveillance reporting [38]. Likewise, there was need to equip the district and health facility levels with trained surveillance personnel in Cameroon [37]. Healthcare personnel identified lack of financial aid as a hindrance to IDSR implementation in Zambia. Hence, necessitating funds allocation in the health sector budget to support IDSR activities [23]. In Nigeria, improved funding would ensure effective surveillance data collection and transmission process [27, 31]. Furthermore, in the South Eastern State of Nigeria, adequate and equitable funding was required to facilitate the disease reporting process [25]. Similarly, increased IDSR funding was recommended to support surveillance activities at the district and health facility levels in Uganda [46]. 


\section{Surveillance attributes}

Key recommendations on improving the surveillance attributes were specific to reporting timeliness and completeness, data quality and accuracy, usefulness, acceptability, simplicity and stability of the surveillance system [20, 22, 30, 33, 34, 36, 37, 41, 42, 44-46].

\section{Timeliness and completeness}

Thirty-three percent (10/30) of the reviewed studies recommended improved reporting timeliness and completeness. An under-performing surveillance quality function requiring improvement was lack of timely reporting within the 24 hour window period for Ebola suspected cases at the regional surveillance unit in Ghana [29]. Similarly, inconsistencies in weekly and monthly reporting timeliness were observed in northern Ghana, hence requiring an urgent need to strengthen the disease surveillance system to enable rapid response to infectious disease outbreaks [22]. Information unavailability on disease notification from health facilities to district level, negated efforts to ascertain surveillance data timeliness in Zimbabwe [34, 41]. Similarly, HCWs in Tanzania responsible for malaria surveillance data reporting were unaware of specific dates when reports were submitted from health facilities to the next reporting level [33]. Therefore, information provision bearing specific reporting dates would be critical to determining surveillance system reporting timeliness. Improved reporting timeliness and completeness in Uganda resulted from enhanced IDSR training, which created increased disease surveillance awareness amongst health providers coupled with mobile-SMS based reporting [46]. Similarly in Malawi, adapting an electronic reporting system and mobile technologies would mitigate disease notification challenges from health facilities to the next level [44]. Furthermore, increased awareness on effective reporting would resolve reporting reluctance amongst health workers and improve reporting timeliness in Nigeria [27]. Reduced workload, enhanced training and improved 
medRxiv preprint doi: https://doi.org/10.1101/2020.09.08.20190553; this version posted September 9, 2020. The copyright holder for this preprint (which was not certified by peer review) is the author/funder, who has granted medRxiv a license to display the preprint in perpetuity.

It is made available under a CC-BY-NC-ND 4.0 International license .

mobile infrastructure would improve reporting completeness and timeliness according to medical and paramedical agents in Madagascar [45]. Moreover, few healthcare providers in South Africa confirmed that the existing notifiable disease surveillance system (NDSS) provided timely information to prompt action. Hence, there were calls for future reforms to give priority to 'timeliness' attribute of South African NDSS to ensure effective disease outbreak containment [20]. Variations in reporting completeness across health system levels in northern Ghana and Cameroon were to be mitigated through undertaking further research investigations to address the root causes and enhancing human resource capacity respectively [22, 37].

\section{Data quality and accuracy}

Four of the 30 studies indicated the need to ensure surveillance data accuracy $[22,34,41,45]$. Scaling-up data entry into the District Health Information Management System (DHIMS2) starting from the health system periphery level would address data quality and accuracy concerns [22]. Erroneous data transmission across surveillance system undermined data quality and surveillance data reliability [45]. Likewise, ensuring missing data in disease notification forms occurred less frequently would improve reported data quality [34, 41]. Moreover, data entries completeness and correctness in notification forms was a data quality measure in Zimbabwe [41].

\section{Simplicity}

Up to $17 \%(5 / 30)$ of the studies required simplification of the surveillance systems $[20,34,36,41$, 45]. Availing easily understandable and simplified terms of reference and case definitions would ease surveillance activities in Madagascar [45]. Notifiable disease surveillance system evaluation in Zimbabwe identified ease and duration of completing disease notification forms as a determinant of system's simplicity $[34,36,41]$. Health workers' positive perceptions on simplicity of the system motivates their involvement in notifying diseases [41]. In contrast, healthcare 
stakeholders involved at operational level of the surveillance system in South Africa perceived the system to be complex compared to their counterparts at health management level. Hence, identifying need for simplifying the system at disease detection and response level [20].

\section{Usefulness}

Two studies in the review alluded to usefulness of existing surveillance systems [20, 41]. There were calls for reforms of South African NDSS to encourage surveillance data use by healthcare providers for outbreak response and communicable diseases control [20]. Besides, effective reports documentation on public health actions or decisions following data collected through disease surveillance systems would be vital in assessing system's usefulness [41].

\section{Acceptability}

Three of the 30 studies gave recommendations on acceptability of the surveillance system [34, 36, 41]. To resolve health workers' lack of willingness to notify diseases in Zimbabwe, there was need for clear designation of surveillance focal persons within health facilities [41]. Similarly, health workers' willingness to participate in surveillance activities was influenced by disease surveillance being in line with their job description $[34,36]$.

\section{Stability}

Sixteen percent (5/30) of the studies identified the need for stable surveillance systems $[30,34,36$, 41, 42]. Enhanced stability of the existing surveillance system in Zimbabwe required an increased number of staff are trained on disease surveillance and provision of functional communication equipment and transport facilities [41]. Similarly, reports on surveillance systems evaluation in Zimbabwe assessed system's stability based on human and material resource availability $[34,36]$. Improved stability of NDSS in South Africa implied the system should be able to provide reliable diagnostic results on notifiable diseases [30]. Furthermore, surveillance system stability in Zambia 
medRxiv preprint doi: https://doi.org/10.1101/2020.09.08.20190553; this version posted September 9, 2020. The copyright holder for this preprint (which was not certified by peer review) is the author/funder, who has granted medRxiv a license to display the preprint in perpetuity.

It is made available under a CC-BY-NC-ND 4.0 International license .

was dependent on internet connectivity consistency or internet outages frequency for a specific time during reporting [42].

\section{Alternative surveillance strategies}

In studies conducted in Zimbabwe, the reporting process was cost intensive due to transport expenses incurred while submitting paper-based disease notification forms. Therefore, electronicbased system adoption for reporting within health facilities would minimise these costs [36, 41]. Disease notifications using the paper-based system for sending notification forms was cost intensive. Hence, requiring the establishment of an electronic-based system for surveillance data reporting and mobile phone technology utilisation [34]. Similarly, there was need to equip health facilities with mobile phone surveillance to effectively capture cholera cases in Cameroon [37]. A suspected Ebola outbreak in Ghana necessitated community-based disease surveillance revival as an active mainstream surveillance system to effectively detect and monitor suspected diseases [29]. In Ethiopia, operational plans formulation for conducting prioritised surveillance visits to specific reporting sites would improve active case searches [43]. To resolve discerned weaknesses in attributes of NDSS in South Africa, it was important to establish an electronic surveillance system utilising mobile phone technology [20]. Similarly, adopting mobile technologies in addition to utilising syndromic surveillance approaches were recommended to strengthen IDSR system in Malawi [44].

\section{Further research on surveillance}

Inconsistencies in surveillance data completeness and timeliness in northern Ghana necessitated further research to mitigate this shortcoming [22]. Limited knowledge amongst health workers on the NDSS coupled with its sub-optimal performance was reported in Zimbabwe [34]. The resolution reached was to initiate further research efforts to assess the effect of health worker 
training on surveillance system performance [34]. In Kenya, further studies to establish reporting challenges facing health facilities in remote settings were recommended [38]. Similarly, there was need to undertake further research in Zimbabwe to ascertain the effect of training health workers on surveillance system aspects [34].

Noteworthy, sub-themes frequency effect size summary based on how often a particular sub-theme appeared in the body of literature reviewed indicated sub-themes relating to knowledge on surveillance systems; technical, material and logistical resources; financial resources and improved surveillance data quality as the predominant emerging sub-themes with frequency effect sizes of $33.3 \%, 20 \%, 20 \%$ and $16.7 \%$ respectively. On the other hand, intensity effect size of studies based on how much each study contributes, in terms of the number of sub-themes it included to the overall body of literature reviewed indicated articles authored by Ngwa et al. 2016 (27.8\%), Nnebue et al. 2013 (27.8\%), Tsitsi et al. 2015 (22.2\%), Lakew et al. 2017 (22.2\%) and Baghdadi, $2016(22.2 \%)$ contributed significantly to the reviewed literature [27, 36, 37, 39, 43]. 


\section{Discussion}

The reviewed assessments studies clearly indicate milestones achieved since adopting the revised IDSR guidelines in Africa, in comparison to findings from a previous review [9]. However, the current review still identifies persistent challenges in IDSR system implementation. This review of recommendations ensuing from prior studies focused on assessing IDSR system functions based on a matrix of major themes inclined to specific surveillance core, support and attribute functions. The recommendations aimed to influence existing health policies by strengthening IDSR system critical functions parallel to reinforcing core surveillance capacities laid out in the IHR [3]. Identifying need to confirm cases is paramount to informing effective and prompt action to avoid disease outbreaks. Therefore, strengthening case confirmation efforts through providing fully functional and adequately equipped laboratory facilities right from the peripheral level is critical for surveillance system effectiveness. In line with our findings, IDSR implementation consolidates surveillance efforts with laboratory support to achieve effective public health action and response [29]. Therefore, improving IDSR strategy performance requires laboratory capacity strengthening to promptly confirm epidemic-prone diseases [29]. However, laboratory capacity challenges still exist in Africa despite the progress made in complying with IDSR and IHR requirements [49]. Weak case confirmation capacities in terms of inadequate laboratory supplies and lack of adequate knowledge among health personnel on specimen handling still exist especially at the peripheral levels $[9,50]$. These limitations affect specimen quality and hinder effective undertaking of case confirmatory tests. Therefore, enhanced laboratory capacity in providing timely collection, transportation and prompt discharge of results is vital to IDSR system functioning in Africa [29].

A key policy challenge relating to laboratory capacity among African countries is lack of 
ownership and consideration of laboratory undertakings and budgets in national health plans [49].

Hence, limiting resource mobilisation and sustainability of laboratory capacity.

Most health systems in African countries especially at peripheral levels rely entirely on paperbased reporting mechanisms due to limited technological and infrastructural capacity [22]. In addition, effective disease surveillance is difficult to achieve in a health system with inadequate infrastructure and a limited health workforce encumbered with surveillance data reporting processes [21]. Therefore, effective surveillance data reporting through correct channels, using appropriate methods and in a timely manner is vital for a well-functioning disease surveillance system. IDSR implementation in Africa is still being confronted with reporting challenges especially at health facility level, which is characterised by limited generation of reliable health information [25]. Reporting forms and guidelines unavailability has an impact on health workers reporting performance and impedes their ability to conduct outbreak investigations [34]. In addition, health workers' awareness on the link between their day-to-day activities and disease reporting will improve their willingness to adhere to reporting guidelines $[25,36]$. Similarly, reporting forms and guidelines availability in health facilities increases disease reporting likelihood [38]. Simplified reporting systems and procedures motivates health workers to report surveillance data effectively as opposed to complex systems [34]. Surveillance data under reporting is associated with severe consequences as result of increased disease burden [51]. Frequent data quality checks through constant supervision would ensure quality surveillance data reporting to the next levels to inform appropriate public health action [34]. Furthermore, reporting forms missing critical information might upset data analysis efforts and further investigations [34]. Hence, the overall surveillance data quality reported dictates public health response quality. 
Feedback is an essential surveillance function and a core IDSR indicator in measuring system's performance [52]. Feedback within a surveillance system involves verbal or written communication from a higher to a lower level. Reviewed studies identified verbal feedback to health facilities as common practice by health personnel usually through half year or quarterly meetings [21, 34, 37]. Further, the review identified a major challenge in feedback mechanisms of existing surveillance systems in Africa, which neglect peripheral levels. Lower surveillance levels are commonly discriminated in receiving epidemiological bulletins from the national levels in comparison to regional surveillance levels [37]. Limited feedback especially at the lower levels have previously been reported, which may demotivate health worker involvement and attitudes towards disease surveillance activities $[50,53]$. Notably, surveillance data forwarding to higher levels with hardly any feedback received at lower levels was commonly practiced prior to revised IDSR guidelines adoption with no clear feedback mechanisms [53, 54]. The review identified recommendations to mitigate inconsistent feedback to lower levels resulting from absence of formal feedback plans and mechanisms as reported by some authors $[53,55]$.

Surveillance data collection, analysis, interpretation and utilisation are intended to inform public health actions [52]. Surveillance data analysis involves morbidity and mortality data aggregation to quantify disease burden at a particular time in a given region. Consequently, this analysis is utilised to monitor disease trends to inform case-based investigations and response [37]. Minimal and basic data analysis is a common practice in health facilities with little or no documented evidence of analysed data [33]. This result from misguided perceptions amongst HCWs on surveillance data generation purposes only for onward reporting rather than utilisation at health facility level [19]. Few health personnel initiated data analysis efforts at health facility level in the African region, with less than fifty percent of surveillance personnel involved in data analysis [19, 
32, 52, 56]. Minimal routine data analysis especially at the lower level facilities has been attributed to limited knowledge and skills among health workers on undertaking analysis of surveillance data and absence of simplified guidelines as suggested by some authors [52, 53, 57].

In line with findings from the review, training of health personnel is linked to strengthened surveillance systems through improved reporting quality and enhanced supervision and feedback across surveillance levels [9]. Moreover, adequate provision of job aids and in-service training on use of reporting forms would improve surveillance system performance $[53,58]$. Low knowledge on the surveillance system amongst HCWs due to infrequent training is considered a key factor affecting IDSR implementation [23]. For instance, lack of trained personnel has a direct effect on efficiency of surveillance functions such as laboratory capacity and the overall performance of surveillance systems [52]. Similarly, training can enhance health worker knowledge on surveillance system, data accuracy and improve reporting timeliness and completeness [21, 22, 59]. However, frequent turnover of trained health staff has a limiting effect on IDSR system optimal functioning [21].

Periodic supervision influences reporting frequency and the quality of surveillance data being reported [21]. From the reviewed studies, it was evident that correct identification of reporting channels was dependent on regular supervision. Therefore, recognising the critical role played by supervision of surveillance activities especially by health workers reporting from the peripheral levels. Surveillance activities frequent and close supervision would enhance utilisation of current information and materials [37]. Hence, ensuring public health actions are well informed by reliable surveillance data rather than outdated information. Supervisory activities lack consistency with efforts mostly initiated during disease outbreaks and this poses a major challenge to achieving 
effective IDSR implementation and performance [42, 46]. Furthermore, strengthened and wellperforming surveillance systems could be achieved through increased supportive supervision by adapting formalized supervisory plans $[9,43]$.

Adequate resource provision facilitates IDSR system optimal functioning. The IDSR strategy was founded on the principle of utilising scarce healthcare resources to effectively achieve disease prevention and control. For instance, reporting forms unavailability may undermine surveillance data quality resulting from incomplete and missing data [60]. The review identified challenges of unavailability of reporting tools, lack of technical guidelines and over dependence on paper-based reporting mechanisms that impede IDSR implementation among Africa countries [22, 37, 42]. Likewise, it was reported in a preceding review that inadequate electronic equipment and unavailability of information, education and communication materials and job aids affected IDSR system performance [9]. The main factors contributing to low quality surveillance data generation are attributed to inadequate funding, limited human resource capacity and unavailability of supporting materials $[35,60]$. Advancements achieved with IDSR system indicators are linked to sustained financial support, hence resulting in IDSR performance stable progression [52]. Similarly, elsewhere, limited budget allocations and delays in disbursement of funds to support surveillance activities hindered effective IDSR implementation [51].

In the pre-adoption phase of revised IDSR guidelines, sensitization and health personnel training would aid improved reporting timeliness and completeness [55, 61]. Similarly, post-adopting the revised IDSR guidelines identified enhanced health worker training as a strategy for improved reporting completeness and timeliness $[45,46]$. However, infrastructural constraints relating to 
logistical and communication systems negatively impact reporting timeliness [62]. Hence, calls for designing and adapting electronic or mobile reporting systems are justified [19, 20, 34, 44, 45, $63,64]$. Unavailability of specific dates for disease notification from health facilities to the next surveillance levels hindered efforts to ascertain reporting timeliness prior to adopting revised IDSR guidelines [61]. Electronic data transfer methods have a direct effect on surveillance data quality [65]. Furthermore, incomplete and unstandardised physical records in health facility registers compromise data accuracy and reliability [9]. Quality control systems incorporation would improve existing surveillance systems' structural capacity to ensure data quality and accuracy are achieved [9].

Evidently, of the reviewed studies, only a few countries in Africa assessed the existing surveillance system considering NTDs. Most of the studies, except for three studies, focused on notifiable conditions that are prioritised within the surveillance systems. For instance, health workers in Madagascar were more aware of case definitions for common conditions such as malaria, diarrhea and respiratory infections compared to other neglected conditions like dengue fever [45]. This low awareness resulted from lack of case definition guidelines, terms of reference and inadequate IDSR training, hence influencing surveillance system's simplicity and applicability to other neglected conditions $[21,45,50]$.

The review further identified pertinent recommendations to achieving improved surveillance performance through influencing health personnel perceptions towards surveillance attributes. Effective disease surveillance systems performance depends on ease of understanding system's functionalities $[34,36,41,66]$. Complex reporting systems tend to demoralise health staff from 
fully participating in case reporting [34]. Perceived surveillance data use in Zimbabwe was mostly intended for resource mobilisation, planning purposes and to a lesser extent for research purposes [36]. On the other hand, perceived surveillance data usefulness was lower amongst healthcare stakeholders responsible for disease detection and response in comparison to those in health management overseeing surveillance activities [20]. Furthermore, timely distribution of information to policy makers and program implementers would be vital to surveillance data usefulness [33]. An acceptable surveillance and response system is well defined by HCWs willingness to voluntarily participate in surveillance activities [14]. Clarity in the designation of health personnel responsible for surveillance data reporting is pertinent to ensuring reporting accuracy and quality control [41]. Additionally, surveillance system stability is dependent on specific factors, which form the bearing of effectively executing surveillance activities. The functioning state of surveillance core and support functions for instance case confirmation, training, human resources, equipment and communication infrastructure may influence surveillance system's stability [30, 34, 36, 41, 42].

Other recommendations in the studies reviewed focused on alternative surveillance strategies. Efforts for active case searching at peripheral levels can be enhanced through establishing wellstructured community based disease surveillance systems [29]. Furthermore, effective active case searching can be achieved through properly designed operational plans targeting priority surveillance areas with high disease reporting sites [43]. Further assessment studies are required in remote settings to strengthen the IDSR system at the peripheral levels bearing higher disease burdens [38]. Similarly, extensive research can identify factors influencing reporting accuracy and inconsistency within surveillance systems [22]. Research efforts initiation to address key 
challenges affecting IDSR system implementation will ensure surveillance system core, support and attribute functions optimal performance in Africa.

Our study had a couple of limitations. First, the review included articles written in English language only, which may have led to some degree of selection bias. Second, the reviewed studies were extracted from only four databases and the review might have missed other studies; however, we believe the search was able to comprehensively capture the surveillance assessment studies conducted in the African region within the selected period. Third, findings were drawn from responses that may have been influenced by social desirability among study participants. Therefore, surveillance assessment studies conducted in future could incorporate observations and document reviews to limit self-reporting bias. Fourth, future reviews could assess and draw lessons on improving IDSR implementation from studies conducted outside the African continent.

\section{Conclusion}

Evidently from this review, consolidated efforts to strengthen all strategic IDSR components is cardinal to achieving effective IDSR strategy implementation in Africa [52]. Notably, findings from the reviewed studies were mostly based on diseases categorised as notifiable conditions within the IDSR framework. Hence, the review identified a critical gap of limited surveillance assessment studies conducted in the African region with a focus on diseases targeted for elimination such as neglected tropical conditions within the IDSR systems. Key recommendations based on health workers' perspectives will inform efforts of prioritising scarce healthcare resources to strengthen surveillance functions of the existing surveillance systems. From the review, it was apparent that most disease surveillance assessment studies conducted in Africa mainly advocated for health worker training [20, 22-27, 29, 34, 36-39, 42, 46]. Training supports 
and cuts across strengthening all surveillance functions. Moreover, knowledge impartment through training on IDSR system functions was considered a critical strategy to ensure disease surveillance system effective functioning. The review identified feedback and reporting as essential surveillance core functions while training, resources and supervision as vital surveillance support functions as perceived by HCWs in Africa. The predominant recommendations regarding surveillance attributes focused on timeliness and completeness. Hence, health policy reviews with a keen focus on strengthening surveillance reporting, feedback, supervision, health worker training, resources and reporting timeliness and completeness would be necessary to achieve effective IDSR system implementation especially at lower surveillance levels. Surveillance functions targeted for immediate improvement within surveillance systems in the African region involve improved reporting, in-service training and increased feedback to lower levels. However, enhanced supervision and resource provision require long-term and sustained support through increased budgetary allocations and refined policies by health ministries. Furthermore, this review depicts systematic reviews as being pragmatic tools to identifying key health system components requiring strengthening. Therefore, it would be pertinent for the WHO Regional Office for Africa in collaboration with national health ministries to constitute health systems review teams to periodically assess surveillance functions performance based on findings emanating from previous surveillance assessment studies to continuously improve IDSR system implementation. This will accurately inform evidence-based efforts to achieving health system strengthening. 


\section{Acknowledgement}

We acknowledge Mrs. Estelle Grobler (Information Specialist) in the Faculty of Health Sciences Library, University of Pretoria for her assistance in the literature search exercise.

\section{Author Contributions}

AKSN: conceived the research idea, developed the proposal, identified the relevant articles for inclusion in the review and screened the titles and abstracts prior to full-text retrieval, quality appraisal and writing the manuscript.

RCK: identified additional articles for inclusion, screening of titles and abstracts, quality appraisal and writing the manuscript.

$\mathrm{CMM}$ and $\mathrm{KV}$ : provided supervision, intellectual input and guidance during proposal development and writing the manuscript.

All the authors read and approved the final version of the manuscript before submission. 


\section{References}

1. Thacker SB, Berkelman RL. Public health surveillance in the United States. Epidemiol Rev. 1988;10:164-90.

2. WHO. Everybody's business: Strengthening health systems to improve health outcomes: WHO's framework for action. Geneva, Switzerland: World Health Organization; 2007.

3. WHO. International Health Regulations (2005): World Health Organization; 2008.

4. Lukwago L, Nanyunja M, Ndayimirije N, Wamala J, Malimbo M, Mbabazi W, et al. The implementation of Integrated Disease Surveillance and Response in Uganda: a review of progress and challenges between 2001 and 2007. Health Policy Plan. 2013;28(1):30-40.

5. WHO. Technical guidelines for integrated disease surveillance and response in the African region. Brazzaville, Republic of Congo and Atlanta, USA. 2010:1-398.

6. Kasolo F, Yoti Z, Bakyaita N, Gaturuku P, Katz R, Fischer JE, et al. IDSR as a Platform for Implementing IHR in African Countries. Biosecurity and Bioterrorism : Biodefense Strategy, Practice, and Science. 2013;11(3):163-9. doi: 10.1089/bsp.2013.0032.

7. Chretien J-P, Lewis SH, editors. Electronic public health surveillance in developing settings: meeting summary. BMC Proc; 2008: BioMed Central.

8. WHO. Integrated Disease Surveillance Quarterly Bulletin. 2016.

9. Phalkey RK, Yamamoto S, Awate P, Marx M. Challenges with the implementation of an Integrated Disease Surveillance and Response (IDSR) system: systematic review of the lessons learned. Health Policy Plan. 2015;30(1):131-43. doi: 10.1093/heapol/czt097 [doi].

10. Sahal N, Reintjes R, Aro AR. Communicable diseases surveillance lessons learned from developed and developing countries: Literature review. Scand J Soc Med. 2009;37(2):187-200.

11. Riesenberg LA, Justice EM. Conducting a successful systematic review of the literature, part 1. Nursing2019. 2014;44(4):13-7.

12. Stern C, Jordan Z, McArthur A. Developing the review question and inclusion criteria. AJN The American Journal of Nursing. 2014;114(4):53-6.

13. WHO. Assessment protocol for national disease surveillance systems and epidemic preparedness and response. Harare, Zimbabwe: WHO Regional Office for Africa, 2000 Contract No.: Report.

14. German RR, Lee LM, Horan JM, Milstein R, Pertowski C, Waller M. Updated guidelines for evaluating public health surveillance systems. MMWR Recomm Rep. 2001;50(1-35).

15. Moher D, Liberati A, Tetzlaff J, Altman DG. Preferred reporting items for systematic reviews and meta-analyses: the PRISMA statement. Ann Intern Med. 2009;151(4):264-9.

16. Popay J, Roberts H, Sowden A, Petticrew M, Arai L, Rodgers M, et al. Guidance on the conduct of narrative synthesis in systematic reviews. A product from the ESRC methods programme Version. 2006;1:b92.

17. Thomas J, Harden A. Methods for the thematic synthesis of qualitative research in systematic reviews. BMC Med Res Methodol. 2008;8(1):45.

18. Dang D, Dearholt SL. Johns Hopkins nursing evidence-based practice: Model and guidelines: Sigma Theta Tau; 2017.

19. Dairo M, Afolayan D, Akinyemi M. Compliance with epidemic-prone diseases surveillance and response guidelines among health officers at surveillance units in South-West Nigeria. Afr Health Sci. 2018;18(2):428-36. doi: https://dx.doi.org/10.4314/ahs.v18i2.28.

20. Benson FG, Musekiwa A, Blumberg L, Rispel LC. Survey of the perceptions of key stakeholders on the attributes of the South African Notifiable Diseases Surveillance System. BMC Public Health. 2016;16(1):1120. doi: 10.1186/s12889-016-3781-7 [doi]. 
21. Adokiya MN, Awoonor-Williams JK, Barau IY, Beiersmann C, Mueller O. Evaluation of the integrated disease surveillance and response system for infectious diseases control in Northern Ghana. BMC Public Health. 2015;15:75-015-1397-y. doi: 10.1186/s12889-015-1397-y [doi].

22. Adokiya MN, Awoonor-Williams J, Beiersmann C, Mueller O. Evaluation of the reporting completeness and timeliness of the integrated disease surveillance and response system in northern Ghana. Ghana Med J. 2016;50(1):3-8.

23. Haakonde T, Lingenda G, Munsanje F, Chishimba K. Assessment of Factors Affecting the Implementation of the Integrated Disease Surveillance and Response in Public Health Care FacilitiesThe Case of Rufunsa District, Zambia. 2018.

24. Lar LA, Afolaranmi TO, Tagurum YO, Uzochukwu B, Zoakah Al. Challenges of integrated disease surveillance response reporting among healthcare personnel in Mangu, Plateau State, Nigeria. Journal of Public Health and Epidemiology. 2015;7(4):108-13.

25. Iwu AC, Diwe KC, Merenu IA, Duru CB, Uwakwe KA. Assessment of disease reporting among health care workers in a South Eastern State, Nigeria. International Journal Of Community Medicine And Public Health. 2016;3(10):2766-74.

26. Nnebue $C$, Onwasigwe $C$, Adogu $P$, Adinma E. Challenges of data collection and disease notification in Anambra State, Nigeria. Tropical Journal of Medical Research. 2014;17(1):1-6. doi: 10.4103/11190388.130173.

27. Nnebue C, Onwasigwe C, Ibeh C, Adogu P. Effectiveness of data collection and information transmission process for disease notification in Anambra State, Nigeria. Niger J Clin Pract. 2013;16(4).

28. Begashaw B, Tesfaye T. Assessment of integrated disease surveillance and response implementation in special health facilities of Dawuro Zone. J Anesthesiol. 2016;4(311):11-5.

29. Issah K, Nartey K, Amoah R, Bachan EG, Aleeba J, Yeetey E, et al. Assessment of the usefulness of integrated disease surveillance and response on suspected ebola cases in the Brong Ahafo Region, Ghana. Infectious diseases of poverty. 2015;4(1):17. doi: 10.1186/s40249-015-0051-3.

30. Benson FG, Musekiwa A, Blumberg L, Rispel LC. Comparing laboratory surveillance with the notifiable diseases surveillance system in South Africa. Int J Infect Dis. 2017;59:141-7. Epub 2017/05/24. doi: 10.1016/j.ijid.2017.03.007. PubMed PMID: 28532981.

31. Ameh CA, Sufiyan MB, Jacob M, Waziri NE, Olayinka AT. Evaluation of the Measles Surveillance System in Kaduna State, Nigeria (2010-2012). Online journal of public health informatics. 2016;8(3):e206. Epub 2017/02/18. doi: 10.5210/ojphi.v8i3.7089. PubMed PMID: 28210427; PubMed Central PMCID: PMCPMC5302462.

32. Abubakar A, Sambo M, Idris S, Sabitu K, Nguku P. Assessment of integrated disease surveillance and response strategy implementation in selected Local Government Areas of Kaduna state. 2013. p. 149.

33. Mboera LEG, Rumisha SF, Mlacha T, Mayala BK, Bwana VM, Shayo EH. Malaria surveillance and use of evidence in planning and decision making in Kilosa District, Tanzania. Tanzania journal of health research. 2017;19(3). doi: 10.4314/thrb.v19i3.7.

34. Maponga BA, Chirundu D, Shambira G, Gombe NT, Tshimanga M, Bangure D. Evaluation of the Notifiable diseases surveillance system in Sanyati district, Zimbabwe, 2010-2011. The Pan African medical journal. 2014;19:278. doi: 10.11604/pamj.2014.19.278.5202 [doi].

35. Adokiya MN, Awoonor-Williams JK, Beiersmann C, Muller O. The integrated disease surveillance and response system in northern Ghana: challenges to the core and support functions. BMC Health Serv Res. 2015;15(Generic):288. Epub 2015/07/29. doi: 10.1186/s12913-015-0960-7. PubMed PMID: 26216356; PubMed Central PMCID: PMCPMC4515924. 
36. Tsitsi JP, Nomagugu N, Gombe NT, Tshimanga M, Donewell B, Mungati M, et al. Evaluation of the Notifiable Diseases Surveillance System in Beitbridge District, Zimbabwe 2015. Open Journal of Epidemiology. 2015;5(03):197.

37. Ngwa MC, Liang S, Mbam LM, Mouhaman A, Teboh A, Brekmo K, et al. Cholera public health surveillance in the Republic of Cameroon-opportunities and challenges. Pan Afr Med J. 2016;24:222. doi: 10.11604/pamj.2016.24.222.8045.

38. Mwatondo AJ, Ng'ang'a Z, Maina C, Makayotto L, Mwangi M, Njeru I, et al. Factors associated with adequate weekly reporting for disease surveillance data among health facilities in Nairobi County, Kenya, 2013. The Pan African medical journal. 2016;23:165. doi: 10.11604/pamj.2016.23.165.8758.

39. Baghdadi I. Assessment of core and support functions of case-based surveillance of meningitis in hospitals in Khartoum State in 2015/Evaluation des fonctions essentielles et des fonctions d'appui du systeme de surveillance des cas de meningite dans les hopitaux de l'Etat de Khartoum en 2015. Eastern Mediterranean Health Journal. 2016;22(4):280.

40. Adokiya MN, Awoonor-Williams JK. Ebola virus disease surveillance and response preparedness in northern Ghana. Global health action. 2016;9(1). doi: 10.3402/gha.v9.29763.

41. Mairosi N, Tshuma C, Juru T, Gombe N, Shambira G, Tshimanga M. Evaluation of Notifiable Disease Surveillance System in Centenary District, Zimbabwe, 2016. Open Journal of Epidemiology. 2017;7(03):251.

42. Mandyata CB, Olowski LK, Mutale W. Challenges of implementing the integrated disease surveillance and response strategy in Zambia: A health worker perspective. BMC Public Health. 2017;17(1). doi: 10.1186/s12889-017-4791-9.

43. Lakew GA, Wassie E, Ademe A, Fenta A, Wube S, Werede M, et al. Status of surveillance and routine immunization performances in Amhara Region, Ethiopia: findings from in-depth peer review. The Pan African medical journal. 2017;27:6. doi: 10.11604/pamj.supp.2017.27.2.10755.

44. Wu TSJ, Kagoli M, Kaasboll JJ, Bjune GA. Integrated Disease Surveillance and Response (IDSR) in Malawi: Implementation gaps and challenges for timely alert. PLoS One. 2018;13(11). doi: 10.1371/journal.pone.0200858. PubMed PMID: WOS:000451763800004.

45. Randriamiarana R, Raminosoa G, Vonjitsara N, Randrianasolo R, Rasamoelina H, Razafimandimby H, et al. Evaluation of the reinforced integrated disease surveillance and response strategy using short message service data transmission in two southern regions of Madagascar, 2014-15. BMC Health Serv Res. 2018;18(1):265.

46. Masiira B, Nakiire L, Kihembo C, Katushabe E, Natseri N, Nabukenya I, et al. Evaluation of integrated disease surveillance and response (IDSR) core and support functions after the revitalisation of IDSR in Uganda from 2012 to 2016. BMC Public Health. 2019;19(1):46. Epub 2019/01/11. doi: 10.1186/s12889-018-6336-2. PubMed PMID: 30626358; PubMed Central PMCID: PMCPMC6327465.

47. Adjei EY, Malm KL, Mensah KN, Sackey SO, Ameme D, Kenu E, et al. Evaluation of cholera surveillance system in Osu Klottey District, Accra, Ghana (2011-2013). Pan Afr Med J. 2017;28:224. Epub 2018/04/10. doi: 10.11604/pamj.2017.28.224.10737. PubMed PMID: 29629010; PubMed Central PMCID: PMCPMC5881562.

48. Jinadu KA, Adebiyi AO, Sekoni OO, Bamgboye EA. Integrated disease surveillance and response strategy for epidemic prone diseases at the primary health care (PHC) level in Oyo State, Nigeria: what do health care workers know and feel? Pan Afr Med J. 2018;31. doi: 10.11604/pamj.2018.31.19.15828. PubMed PMID: WOS:000454296200001.

49. Yahaya A, Ndihokubwayo J, Coulibaly S, Akanmori B, Mwenda J, Dosseh A. Laboratory capacity in 2012 for diagnosis of epidemic prone diseases in the context of Integrated Disease Surveillance and Response in the WHO African Region. African Health Monitor. 2013;(18):44-8. 
50. Nsubuga P, Brown WG, Groseclose SL, Ahadzie L, Talisuna AO, Mmbuji P, et al. Implementing Integrated Disease Surveillance and Response: Four African countries' experience, 1998-2005. Global public health. 2010;5(4):364-80. doi: 10.1080/17441690903334943 [doi].

51. Dairo M, Bamidele J, Adebimpe W. Disease surveillance and reporting in two Southwestern states in Nigeria: Logistic challenges and prospects. Journal of public health and Epidemiology. 2010;2(6):1259.

52. Lukwago L, Nanyunja M, Ndayimirije N, Wamala J, Malimbo M, Mbabazi W, et al. The implementation of Integrated Disease Surveillance and Response in Uganda: a review of progress and challenges between 2001 and 2007. Health Policy Plan. 2013;28(1):30-40. doi: 10.1093/heapol/czs022. PubMed PMID: WOS:000313655100004.

53. Mghamba JM, Mboera LEG, Krekamoo W, Senkoro KP, Rumisha SF, Shayo E, et al. Challenges of implementing an integrated disease surveillance and response strategy using the current health management information system in Tanzania. Tanzania journal of health research. 2004;6(2):57-63.

54. Mboera L, Rumisha S, Magesa S, Kitua A. Utilisation of health management information system in disease surveillance in Tanzania. Tanzania journal of health research. 2001;3(2):15-7.

55. Franco LM, Fields R, Mmbuji PK, Posner S, Mboera LE. Situation analysis of infectious disease surveillance in two districts in Tanzania 2002. 2003.

56. Gueye D, Senkoro KP, Rumisha SF. Baseline monitoring and evaluation of integrated disease surveillance and response in Tanzania. 2005.

57. Abubakar AA, Idris SH, Sabitu K, Shehu AU, Sambo MN. Emergency preparedness and the capability to identify outbreaks: A case study of Sabon Gari Local Government Area, Kaduna state. Annals of Nigerian Medicine. 2010;4(1):21.

58. Gueye D, Banke K, Mmbuji P. Follow-Up Monitoring and Evaluation of Integrated Disease Surveillance and Response in Tanzania: Partners for Health Reformplus; 2006.

59. Bawa SB, Olumide EA. The effect of training on the reporting of notifiable diseases among health workers in Yobe State, Nigeria. Niger Postgrad Med J. 2005;12(1):1-5. Epub 2005/04/14. PubMed PMID: 15827587.

60. Phalkey RK, Shukla S, Shardul S, Ashtekar N, Valsa S, Awate P, et al. Assessment of the core and support functions of the Integrated Disease Surveillance system in Maharashtra, India. BMC Public Health. 2013;13(1):575. doi: 10.1186/1471-2458-13-575.

61. Pond B, El Sakka H, Wamala J, Lukwago L. Mid-term evaluation of the integrated disease surveillance and response project. Washington, DC: USAID. 2011.

62. Mboera LE, Rumisha SF, Mwanemile EJ, Mziwanda E, Mmbuji PK. Enhancing disease surveillance reporting using public transport in Dodoma District, Central Tanzania. Tanzan Health Res Bull. 2005;7(3):201-5.

63. Mtema Z, Changalucha J, Cleaveland S, Elias M, Ferguson HM, Halliday JE, et al. Mobile phones as surveillance tools: implementing and evaluating a large-scale intersectoral surveillance system for rabies in Tanzania. PLoS Med. 2016;13(4):e1002002.

64. Kiberu VM, Matovu JK, Makumbi F, Kyozira C, Mukooyo E, Wanyenze RK. Strengthening districtbased health reporting through the district health management information software system: the Ugandan experience. BMC Med Inform Decis Mak. 2014;14:40-6947-14-40. doi: 10.1186/1472-694714-40 [doi].

65. Awoonor-Williams JK, Bawah AA, Nyonator FK, Asuru R, Oduro A, Ofosu A, et al. The Ghana essential health interventions program: a plausibility trial of the impact of health systems strengthening on maternal \& child survival. BMC Health Serv Res. 2013;13(2):S3.

66. Mutale W, Chintu N, Amoroso C, Awoonor-Williams K, Phillips J, Baynes C, et al. Improving health information systems for decision making across five sub-Saharan African countries: implementation strategies from the African Health Initiative. BMC Health Serv Res. 2013;13(2):S9. 